\title{
The association of sensory phenotype and concomitant mood, sleep and functional impairment with the outcome of carpal tunnel surgery
}

Donna L. Kennedy ${ }^{1,2^{*}}$, Deborah Ridout ${ }^{3}$, Ladislava Lysakova ${ }^{4}$, Jan Vollert ${ }^{1,5,6,7}$, Caroline M. Alexander ${ }^{2,8}$ and Andrew S. C. Rice ${ }^{1}$

\begin{abstract}
Background: Up to $25 \%$ of people who have had carpal tunnel release surgery (CTR) fail to report improvement; however, evidence for prognostic indicators in this surgical cohort is limited. To identify candidate prognostic factors, this study investigated the association of quantitative sensory testing (QST) derived sensory phenotype and attendant impairment with patient-reported surgical outcome.

Methods: With ethical approval and informed consent, this prospective observational longitudinal study recruited patients from two London hospitals. Multimodal phenotyping measures including quantitative sensory testing (QST), pain parameters, insomnia, pain-related worry, mood and function, were evaluated prior to; and at 3-and 6-months post-surgery. Pain in median nerve distribution with electrophysiologically confirmed conduction delay and DN4 score $\geq 4$ was defined as neuropathic. Primary outcome was patient-rated change at 6 months, dichotomised as poor outcome; "worse" or "no change" and good outcome; "slightly better", "much better" or "completely cured".

Results: Seventy-six patients participated. Prior to surgery, substantial heterogeneity in established categories of somatosensory function was observed with $21 \%$ of participants categorised as having a healthy sensory phenotype; $29 \%$ with thermal hyperalgesia; $32 \%$ mechanical hyperalgesia and $18 \%$ sensory loss. Seventy six percent of participants were classified as having neuropathic pain, $33 \%$ with high levels of pain related worry and $64 \%$ with clinical insomnia. Observed differences in pain, sleep impairment, psychological factors and function, between sensory phenotypic groups, was not significant. At 3-and 6-months post-surgery there was significant improvement in all phenotyping measures with a moderate to large effect size. Thermal and mechanical measures of somatosensation improved ( $p<0.001)$, as did functional ability $(p<0.001)$. Symptom severity diminished $(p<0.001)$, as did pain-related worry $(p<0.001)$, anxiety $(p=0.02)$ and insomnia $(p<0.001)$. Patient-rated surgical outcome was good in $92 \%$ of the cohort, poor in $8 \%$. Baseline sensory phenotype category was not associated with surgical outcome however painrelated worry, anxiety and functional interference were significantly associated with outcome $(p \leq 0.05)$.
\end{abstract}

\footnotetext{
*Correspondence: d.kennedy@imperial.ac.uk

${ }^{1}$ Pain Research, Department of Surgery and Cancer, Faculty of Medicine,

Chelsea \& Westminster Hospital Campus, Imperial College London, 369

Fulham Rd, London SW10 9NH, UK

Full list of author information is available at the end of the article
}

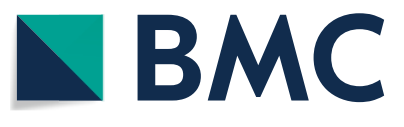

(c) The Author(s) 2021. Open Access This article is licensed under a Creative Commons Attribution 4.0 International License, which permits use, sharing, adaptation, distribution and reproduction in any medium or format, as long as you give appropriate credit to the original author(s) and the source, provide a link to the Creative Commons licence, and indicate if changes were made. The images or other third party material in this article are included in the article's Creative Commons licence, unless indicated otherwise in a credit line to the material. If material is not included in the article's Creative Commons licence and your intended use is not permitted by statutory regulation or exceeds the permitted use, you will need to obtain permission directly from the copyright holder. To view a copy of this licence, visit http://creativecommons.org/licenses/by/4.0/. The Creative Commons Public Domain Dedication waiver (http://creativeco mmons.org/publicdomain/zero/1.0/) applies to the data made available in this article, unless otherwise stated in a credit line to the data. 
Conclusion: In patients undergoing carpal tunnel surgery, pain-related worry, anxiety and pain functional interference are candidate prognostic outcome factors and require further elucidation.

Keywords: Anxiety, Carpal tunnel surgery, Catastrophizing, Insomnia, Neuropathic pain, Phenotype, Quantitative sensory testing (QST)

\section{Background}

Carpal tunnel syndrome (CTS), compression of the median nerve at the wrist, is the most prevalent of the entrapment neuropathies [1]. Prevalence estimates vary based on diagnostic criteria; however, it is estimated that one in 10 people will develop carpal tunnel syndrome at some point [2]. CTS symptoms include pain, paraesthesiae and/or numbness in the median nerve distribution of the hand and weakness of the thenar muscles. Symptoms impair performance of daily activities and adversely affect quality of life. Median nerve decompression surgery, or carpal tunnel release (CTR), is an efficacious treatment for this potentially debilitating condition and is the most commonly performed procedure in the hand $[3,4]$. However, carpal tunnel surgery is not without risk of adverse events $[5,6]$ and significantly, up to $25 \%$ of patients fail to report improvement following surgery $[7,8]$.

Clearly, it would be advantageous if clinicians, prior to surgery, could anticipate treatment response for a given patient. This might underpin a personalised medicine approach, guiding patient stratification, modification in care pathways or enabling the use of 'prehabilitation' approaches to surgery preparedness [9]. However, at present, there is limited evidence for prognostic factors associated with CTR outcome. The severity of electrophysiologically assessed nerve conduction delay has been investigated extensively, but findings from observational studies are contradictory and inconclusive [10-20]. Studies employing a prognostic factor design [21] have identified that greater functional impairment [22], increased utilisation of health care resources, a greater number of comorbid health conditions and higher levels of anxiety [23] prior to surgery are prognostic factors associated with poorer surgical outcome. Consistent with these findings, a review of the CTR outcome literature concluded that poorer general health and comorbid conditions including diabetes, alcohol consumption and smoking were associated with a worse surgical prognosis [19].

While pain is prevalent in CTS [2] and is neuropathic in nature in up to $80 \%$ of patients $[24,25]$, the mechanistic nature of pain in CTS has not been widely studied nor fully elucidated [26, 27]. It is recognised that for patients with neuropathy, pain is heterogeneous, manifesting diversely across those with a common clinical condition [28]. An individual's profile of sensory symptoms and signs, or sensory phenotype [29], is thought to reflect this inter-individual heterogeneity in underlying pathophysiology of pain pathways and mechanisms in neuropathic pain conditions [30-33].

It is acknowledged that neuropathic pain, caused by a lesion or disease of the somatosensory nervous system $[34,35]$ poses a considerable, multidimensional burden for patients [36]. Secondarily, consensus group evaluation guidelines are underpinned by the biopsychosocial model of pain, consistent with the International Classification of Functioning, Disability and Health [37]. Accordingly, in addition to pain parameters, the domains of mood, sleep and function are integral elements of a patient-centred evaluation $[38,39]$ and resonate with patient priorities [40].

Sensory phenotype has previously been described in patients with carpal tunnel syndrome, demonstrating heterogeneity in small and large median nerve fibre somatosensory dysfunction $[25,41]$, however it is not clear if sensory phenotype is associated with the outcome of carpal tunnel surgery. Furthermore, it is not known if attendant burden in the domains of pain, mood, sleep interference and functional impairment differs between sensory phenotypic groups, or if these domains are prognostic factors associated with surgical outcome. Identifying intra-individual factors associated with patient-reported symptom severity and surgical outcome may inform stratified care for patients with carpal tunnel syndrome, better guiding treatment decisions and thereby improving outcomes.

We aimed to investigate the association of sensory phenotype and concomitant pain parameters, psychological factors, sleep restriction and functional impairment with outcome at 6 months post carpal tunnel surgery in order to identify candidate prognostic factors for future investigation. Secondarily, we aimed to explore heterogeneity in sensory phenotype and associated comorbidity in the domains of pain, mood, sleep and function in order to establish whether symptom clusters are identifiable within the population of patients with carpal tunnel syndrome. The hypothesis being, in patients with carpal tunnel syndrome, there is a constellation of neuropathic pain associated clinical features which predispose this phenotypic group to symptoms which do not improve or worsen following surgery. 


\section{Methods}

Ethical approval was granted by the Camberwell St Giles National Research Ethics Committee (14/LO/1436) on 29 August 2014 for a prospective, longitudinal observational study. Two patient-collaborators (SP; AT), previewed the study measures and procedures and edited study documents for clarity and acceptability. Sequential adult patients listed for open carpal tunnel decompression surgery at two London National Health Service (NHS) hospitals were recruited by poster and in person at their hospital clinic appointment and by post. A comparable surgical technique was used at the two recruitment sites; open decompression with a median nerve block under tourniquet control. Incisions were closed with nonabsorbable sutures. Participants were not paid for study participation however travel was reimbursed.

\section{Participation criteria}

Exclusion criteria were significant cognitive dysfunction, patient-defined lack of English language adequate for completing study questionnaires and participating in psychophysical testing, a history of potentially confounding conditions (rheumatoid arthritis, renal failure, peripheral neuropathy of any origin other than CTS), steroid injection of the study limb within the previous 4 weeks or previous carpal tunnel surgical release in the study hand, anatomic abnormalities of the wrist or hand, median nerve injury or compression secondary to traumatic injury and pregnancy.

\section{Sample size}

Participants were stratified by sensory phenotype using the German Research Network on Neuropathic Pain (DFNS) quantitative sensory testing (QST) protocol [42] and algorithm [43]. Published age and sex stratified QST reference data for the hand pertain to the dorsal, radial nerve innervated hand [44] and are not generalisable to the volar, median nerve innervated hand [25]. Therefore, to identify sensory dysfunction in this CTS cohort, sample size was calculated based on an independent samples t-test to determine a difference in cold pain threshold (CPT), as evaluated with the DFNS QST protocol, for patients with carpal tunnel syndrome tested at the volar middle finger (mean $17.3 ; \pm 5.9^{\circ} \mathrm{C}$ ) and healthy controls (mean $13.4 \pm 7.5^{\circ} \mathrm{C}$ ) [45]. Control data was generated from a convenience sample of participants in a previously reported healthy volunteer quantitative sensory testing study [46]. Of the thirteen QST measures in the DFNS battery, CPT was chosen as a measure of importance because in other cohorts, cold pain sensitivity has been demonstrated to be associated with pain and disability outcomes [47] and surgical outcome [48]. A minimum sample size of 94 (47 CTS participants and 47 healthy controls) was required to achieve a power of $80 \%$ and a level of significance of $5 \%$ (two sided), for detecting a difference of a similar size for QST.

\section{Surgical outcome}

The measure used to categorize surgical outcome as good or poor (binary outcome) was a patient-reported global rating of change (PGRC) at 6 months post-surgery. The PGRC is a 5 -point ordinal scale whereby $1=$ worse; $2=$ unchanged; $3=$ slightly better; $4=$ much better and $5=$ completed cured $[7,18,49,50]$ with a grade of 3 or above interpreted as a good outcome or treatment success. Where investigators [7, 49] have defined treatment success as 4 or above using the same ordinal scale, they note that their patients are selected for surgery based on a good prognosis, therefore their findings are less generalizable to the wider population of patients. However, in a comparable pragmatic prospective cohort including participants with multiple comorbidities, a grade of 3 (slightly better) was similarly identified as representative of treatment success [50]. Other surgical outcome measures included patient rated symptom severity and functional impairment evaluated with the Boston Carpal Tunnel Questionnaire (BCTQ) [51] and post-surgical scar pain and interference [52]. Participants sealed completed outcome measures in pre-labeled, coded envelopes which were secured in the written case report form thereby blinding the investigator (D.K.) until participants completed the trial.

\section{Procedure}

Median nerve somatosensory function, pain parameters, psychological state and quality of life measures were evaluated prior to and at 3- and 6-months post-surgery. Baseline measures were completed within 6 weeks prior to surgery; 3- and 6-months post- surgery assessments were completed within \pm 21 days. Where participants failed to attend a 3- or 6-month assessment, outcome questionnaires were posted. At baseline, demographic data and medical history was recorded. Clinical history taking utilised the Sangha Comorbidity Questionnaire, a patient-reported tool validated for quantifying comorbid conditions and their impact on function, thereby enabling the comparison of general health between participants [53]. Comorbidity scores range from 0 to 45, with higher scores implying poorer general health and functional impairment. All tests and questionnaires were delivered in the same order across the participants, across visits.

\section{Definition of neuropathic pain}

Nerve conduction studies (NCS) were performed by the respective hospital neurophysiology departments and severity graded according to Bland [54] criteria. Pain was 
categorised as neuropathic where there was neurophysiologic confirmation of median nerve conduction delay; a score of $\geq 4$ on the Douleur Neuropathique 4 questions (DN4) and where pain was present in the median nerve distribution as reported on a participant completed pain map [34, 38, 55]. Post-surgery this two-stage triage was repeated however, in lieu of repeat electrophysiological testing, two or more abnormal QST findings indicative of loss of sensory function was taken as a confirmatory diagnostic test for neuropathic pain.

\section{Multimodal Phenotyping measures}

Relevant phenotypic characteristics and measures were derived from the Initiative on Methods, Measurement, and Pain Assessment in Clinical Trials (IMMPACT) consensus guidelines for patient phenotyping in clinical trials of pain treatments [56], commensurate with consensus guidelines for the assessment of patients with neuropathic pain [38]. Phenotypic characteristics of interest included quantitative sensory testing (QST) derived sensory phenotype, pain parameters, psychological factors including catastrophic thinking in relation to pain, anxiety and depression, sleep restriction and functional impairment.

\section{Sensory phenotype}

Somatosensory function was evaluated according to the German Research Network on Neuropathic Pain (DFNS) quantitative sensory testing (QST) protocol [42]; indepth methods have been previously reported elsewhere [25]. All equipment was calibrated prior to testing. In brief, thermal detection thresholds, thermal pain thresholds and thermal sensory limen were evaluated with a Somedic MSA thermal stimulator (Sweden) with an $18 \mathrm{~mm}^{2}$ metal Somedic thermode. Mechanical detection threshold was tested using glass monofilaments (Optihair2-Set, Marstock Nervtest, Germany) with bending forces between 0.25 and $512 \mathrm{mN}$. Mechanical pain threshold, mechanical pain sensitivity and windup ratio were evaluated using blunt probes with forces ranging from 8 to $512 \mathrm{mN}$ (pinprick stimulator, MRC, Heidelberg, Germany). Dynamic mechanical allodynia was tested with a cotton wisp, a cotton bud (Q-Tip) and a standardised brush designed to produce minimum friction (Somedic, Sweden). Vibration detection threshold testing used a Rydel-Seiffer graded tuning fork $(64 \mathrm{~Hz}$, $8 / 8$ scale). Pressure pain threshold was tested with a pressure algometer (FDN100, Wagner Instruments, Greenwich, CT, USA) with a surface area of $1 \mathrm{~cm}^{2}$ and by applying pressure at a rate of $1 \mathrm{~kg} / \mathrm{cm}^{2}$ per second. Pressure pain threshold was tested at the thenar eminence, all other tests were performed at the volar distal phalanx of the middle finger.

Using the DFNS algorithm [43], participants were categorised as having a "healthy" sensory phenotype or stratified to a thermal hyperalgesia, mechanical hyperalgesia or sensory loss phenotype (Fig. 1) [57]. In the context of testing patients with CTS, a "healthy" sensory phenotype suggests that sensory function is not characterised by small fibre dysfunction, as would be consistent with neuropathy.

\section{Pain parameters}

Pain symptoms, signs and descriptors were assessed with the Douleur Neuropathique 4 questions (DN4) [58]. The DN4 consists of seven symptom questions and three sensory examination measures; a score of $\geq 4$ is considered diagnostic of neuropathic pain. Pain dimensions were assessed with the Neuropathic Pain Symptom Inventory (NPSI) [59], a validated patient-completed

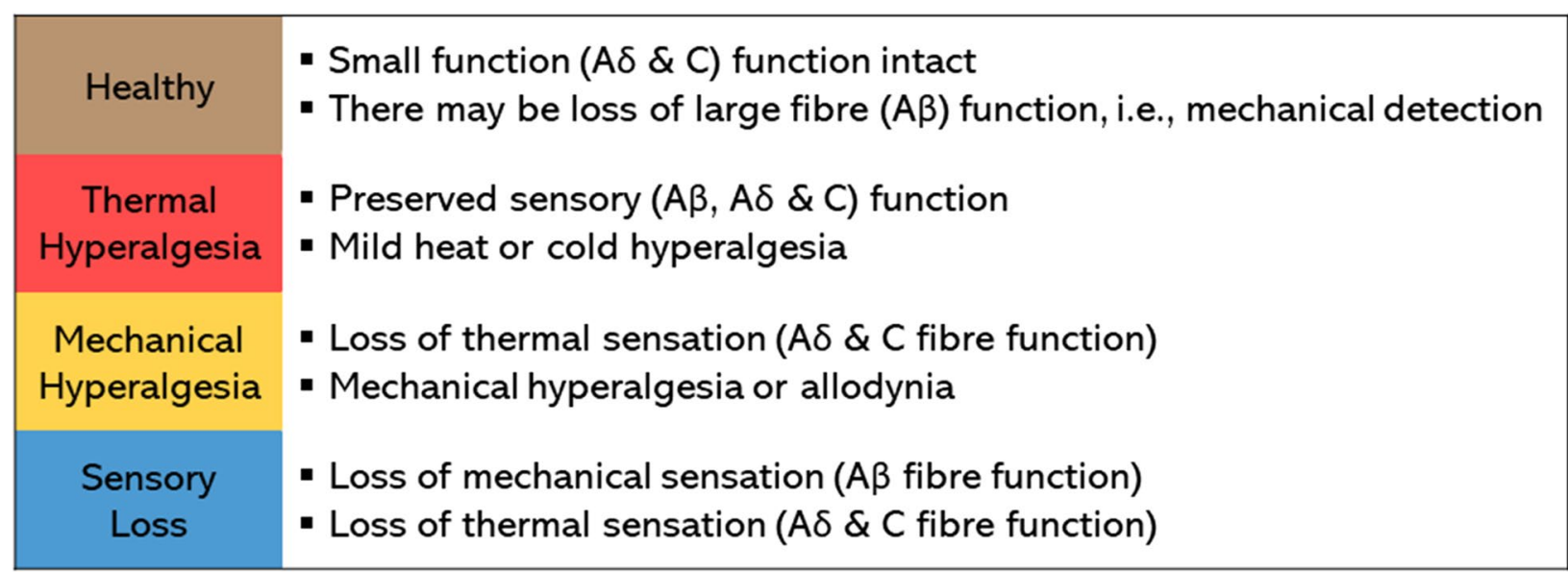

Fig. 1 Quantitative sensory testing (QST) derived sensory phenotypes [43] and associated sensory impairment characteristics 
inventory for evaluating the nature of neuropathic pain, including spontaneous, paroxysmal and evoked pain and paraesthesia/dysesthesia. Total NPSI scores range from 0 to 100 with greater scores implying more severe symptom severity; item scores of 1-3 indicate mild pain severity, 4-6 moderate and 7-10 severe [60]. Pain severity and interference was assessed with the validated [61] Brief Pain Inventory (BPI) [62]. The BPI is a twopart questionnaire whereby a Pain Severity Score (PSS) is calculated as the mean of four questions quantifying present pain and the least, worst, and average pain over the last week. Pain is rated on an 11-point scale ranging from 0 (no pain) to 10 (pain as bad as you can imagine). There are no universally accepted cut-points for interpreting pain scales however in patients with chronic musculoskeletal pain, ratings of $1-3$ out of 10 are suggested to correspond with mild pain, 4-6 with moderate pain and 7-10 severe pain $[63,64]$. Pain severity and frequency was assessed with the Boston Carpal Tunnel Questionnaire (BCTQ) Symptom Severity Scale (SSS) [51], a CTS specific, patient-completed questionnaire which is well validated, reliable and responsive $[65,66]$. Eleven symptoms are rated on a 5-point scale with lower scores implying milder symptoms.

\section{Psychological factors}

Pain-related worry was evaluated with the Pain Catastrophizing Scale (PCS) [67]. Pain catastrophising is defined as exaggerated, persistent thought related to painful experiences coupled with a perceived inability to cope with such experiences $[67,68]$. However, at present, questions have arisen as to the validity of assessing pain catastrophizing based on self-report measures; it is proposed the construct being evaluated with self-report measures is more appropriately described as 'pain-related worry' [69]. The PCS yields a total score (range 0-52) and three subscale scores assessing rumination (range 0-16), magnification (range 0-12) and helplessness (range $0-24)$. A PCS score of 30 or greater is described as indicative of clinically relevant pain-related worry. Mood was assessed with the Depression, Anxiety and Positive Outlook Scale (DAPOS) [70] an 11-item questionnaire. The DAPOS was developed and validated specifically for use in patients with chronic pain. The DAPOS has 3 independent scales; depression, anxiety and positive outlook. Scores for the subscales range from 5 to 25 for depression, 3 to 15 for anxiety, and 3 to 15 for positive outlook. There is no total score for the DAPOS nor a defined score for clinically relevant depression or anxiety [71].

\section{Sleep}

Sleep interference was evaluated with the Insomnia Severity Index (ISI) [72], a 7 item measure (score range
0-28) that quantifies sleep disturbance and the impact of insomnia on function and quality of life. Scores of 7 or less are interpreted as suggesting no clinically significant insomnia; 8-14 as subthreshold insomnia; $15-21$ as clinical insomnia of moderate severity and $22-28$ as severe clinical insomnia.

\section{Functional impairment}

Functional impairment was evaluated with the patientcompleted Boston Carpal Tunnel Questionnaire (BCTQ) [51] 8 item Functional Status Scale (FSS). Items are rated on a 5 -point scale with lower scores imply milder symptoms and less functional impairment. Additionally, pain interference was assessed with the Brief Pain Inventory (Short Form) (BPI-SF) Interference Scale [62]. Seven BPI-SF items are scored on an 11-point scale ranging from 0 (does not interfere) to 10 (completely interferes), quantifying pain interference in general activity, walking, work, relationships, mood, life enjoyment, and sleep and reported as the mean of the seven items.

\section{Statistical analysis}

All continuous data were tested for normality of distribution. To categorise sensory phenotype, QST results were compared to control data generated from a convenience sample of healthy volunteers [46]. Z-scores were calculated ( $z=$ [value of participant - mean value of controls] / standard deviation of controls) [44]. Values outside the range of $\mathrm{z} x \pm 1.96$ were interpreted as abnormal, positive $\mathrm{z}$ scores denote a gain in function (hyperalgesia) whereas negative scores indicate a loss of function.

Patient characteristics and distribution of phenotyping measures were summarized using descriptive statistics. To identify differences in attendant burden (i.e., pain, mood, sleep impairment, functional impairment) between sensory phenotypic groups, differences in phenotyping measures were analysed with the non-parametric Kruskal-Wallis Test; comparison of pairs was conducted with the Mann-Whitney U Test (actual, not corrected $P$ values reported).

Change in multimodal phenotyping measures across three time points (baseline; 3 months; 6 months) was investigated with one-way repeated measures analysis of variance (ANOVA) with Bonferroni correction and pairwise comparisons or the non-parametric Friedman test, as appropriate. Effect size was investigated with partial eta squared [73] and interpreted as $0.01=$ small, $.05=$ medium and $0.14=$ large [74].

The primary outcome, patient completed global rating of change (PGRC) at 6 months post-surgery was used to classify surgical outcome as a binary variable; a good versus poor outcome. A score of 3 or better was interpreted as a good outcome, or treatment success. Chi-square test 
for independence was used to investigate the relationship between sensory phenotype and patient-reported surgical outcome; significance is reported for the Pearson ChiSquare value or Fisher's Exact Probability Test where cell counts were less than 5 . Whereas the analysis plan was to explore the relationship between baseline multimodal phenotyping measures (pain parameters, psychological factors, sleep restriction and functional impairment) with carpal tunnel surgery outcome (PGRC) using multivariate analysis of variance, this was precluded by the small number of participants in the "poor surgical outcome" group. Therefore, the association of phenotyping measures and surgical outcome was investigated with the Spearman Rank Order Correlation Coefficients. Where correlation coefficients were statistically significant $(p \leq .05)$, the strength of relationship was interpreted as small $r=.10$ to .29 ; medium $r=.30$ to .49 ; large $r=.50$ to 1.0 [74].

\section{Results}

Seventy-six participants were enrolled between October 2014 and December 2016 and completed baseline study measures; however, 4 participants did not undergo surgery (one patient declined surgery and in 3 cases surgery was cancelled due to ongoing medical investigations) (Fig. 2). Demographic data and health parameters for the sample are reported in Table 1 . The sample was comprised predominantly of white females who were currently employed and undergoing surgery on their dominant hand $(n=53 ; 70 \%)$. In the majority of
Table 1 Key demographic and health parameters

\begin{tabular}{ll}
\hline Age mean years (SD) & $58.5(13.5)$ \\
Female sex n (\%) & $65(86)$ \\
Body mass index mean (SD) & $28.8(6.8)$ \\
Comorbidity score mean (SD) & $5.1(4.0)$ \\
Symptom duration mean months (SD) & $52(48)$ \\
Smoking history n (\%) & \\
$\quad$ Never smoked & $30(39)$ \\
Previous smoker & $35(46)$ \\
Present smoker & $11(15)$ \\
Ethnicity n (\%) & \\
White & $48(64)$ \\
Asian & $11(15)$ \\
Black & $12(16)$ \\
Mixed & $4(5)$ \\
Arab & $1(1)$ \\
Profession n (\%) & \\
Manual; service trades & $29(38)$ \\
Administrative \& technical & $17(22)$ \\
Professionals & $30(39)$ \\
Employment history n (\%) & \\
Employed & $42(55)$ \\
Unemployed & $12(16)$ \\
Retired & $22(29)$ \\
\hline
\end{tabular}

Key: Standard deviation (SD)

participants (76\%), severity of nerve compression was graded from moderately to extremely severe (very mild $4 \%$; mild $21 \%$ moderately severe $29 \%$; severe $18 \%$; very severe $21 \%$; extremely severe $3 \%$ ) [54].

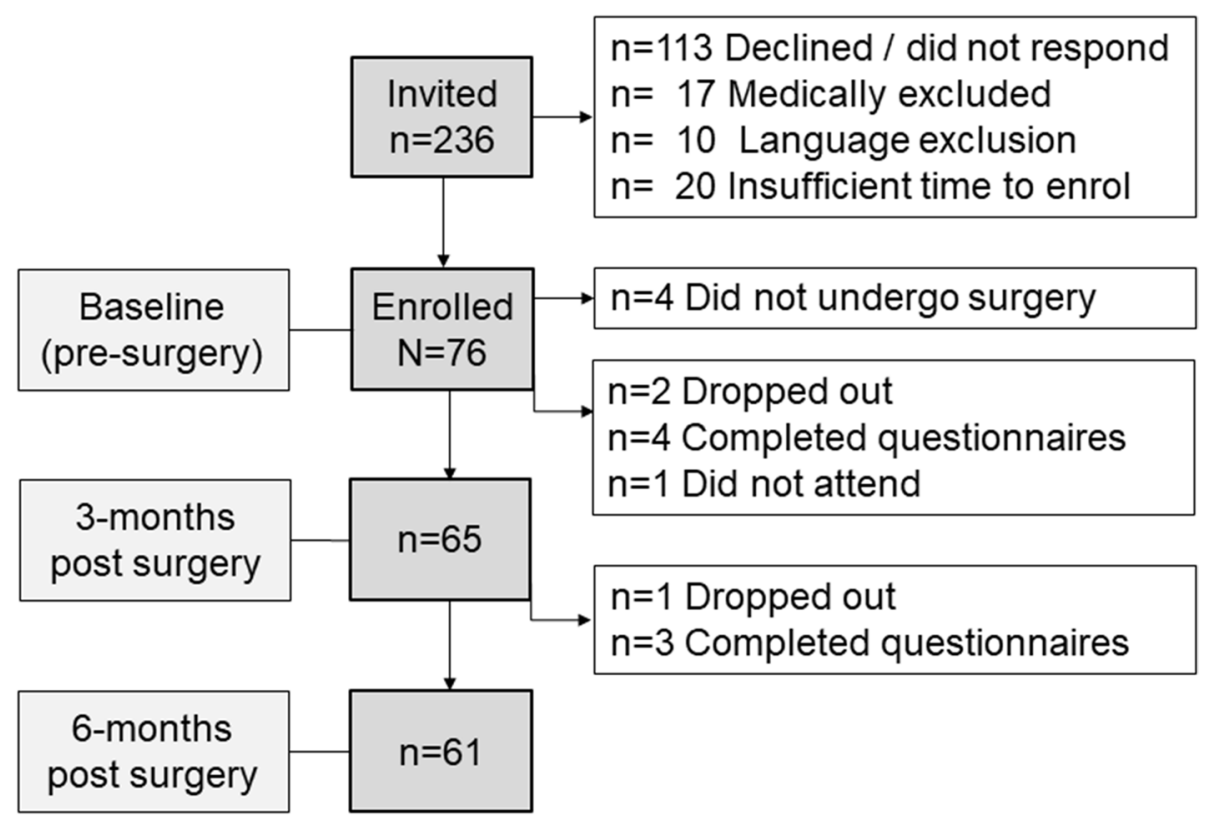

Fig. 2 Study recruitment and enrolment 


\section{Sensory phenotype}

To derive sensory phenotype, QST results were compared to control data from a previously reported convenience sample of 54 healthy volunteers [mean age 54.9 years (standard deviation 11.3); 38 (70\%) female] [25]. At baseline, 16 (21\%) of participants were defined as having a "healthy" sensory profile, 22 (29\%) thermal hyperalgesia, 24 (32\%) mechanical hyperalgesia and 14 (18\%) sensory loss phenotype. Change in sensory phenotype from baseline to 3 and 6-month post-surgical assessments was statistically significant $(p<.001)$ (Fig. 3$)$.

QST results, priority to surgery, clearly illustrate this marked heterogeneity in somatosensory function. Loss of small fibre function (thermal detection and cold hyperalgesia) was observed in up to $25 \%$ of patients (Fig. 4); whereas paradoxical heat sensations, a pathological response, were rarely observed (pre-surgery $14 \%$; 3 months post-surgery $17 \%$; 6 months post-surgery $10 \%)$. Loss of large fibre function (mechanical detection threshold and vibration detection threshold) was the predominant sensory feature, identified in up to $60 \%$ of participants. In contrast, mechanical hyperalgesia, as evidenced by decreased mechanical pain threshold and increased mechanical pain sensitivity, was only observed in 16\% of the cohort (Fig. 5). Finally, dynamic mechanical allodynia, a pathological sensory response, was not exhibited by CTS participants prior to or following surgery.

\section{Pain parameters}

All pain parameters were normally distributed. At baseline, $76 \%$ of participants reported pain that was categorised as neuropathic in nature. Change in DN4 scores were statistically significant from baseline to 3 months and baseline to 6 months $(p<.001)$ (Table 2$)$. Of the 32 participants who continued to report pain at 6 months post-surgery, in $24(38 \%)$ this was mild, $8(13 \%)$ moderate and $3(5 \%)$ severe [63, 64].

For all pain parameters, there was a significant effect for time $(p<.001)$ and the magnitude of the effect size was large, demonstrating significant improvement in pain post-surgery. Differences in baseline pain parameters, between sensory phenotypic groups, were explored. In addition, baseline sensory phenotype was used to investigate if there were observable differences in pain trajectories between sensory phenotypic groups at 6 months post-surgery (Table 3). For all pain parameters, both presurgery and at 6 months post-surgery, burden is lowest in participants with a healthy sensory phenotype and highest in those with a sensory loss phenotype. While baseline between-group differences were not statistically significant (Kruskal-Wallis Test $p>.05$ ), differences at 6 months approached significance for the Symptom Severity Scale score and were significant for the NPSI $(p=0.04)$.

\section{Pain-related worry}

At baseline, 25 (33\%) of participants presented with clinically relevant pain-related worry based on Pain Catastrophizing Scale (PCS) scores; at 3 months this was reduced to $7(11 \%)$ and at 6 months 8 (13\%) (Fig. 6). Change in total PCS score from baseline (mean 20.08, standard deviation 13.36) to 3 months (mean 11.02, standard deviation 12.37) and baseline to 6 months (mean 10.36, standard deviation $12.39)$ was statistically significant $(p<.001)$. Differences in the magnitude of pain-related worry between sensory

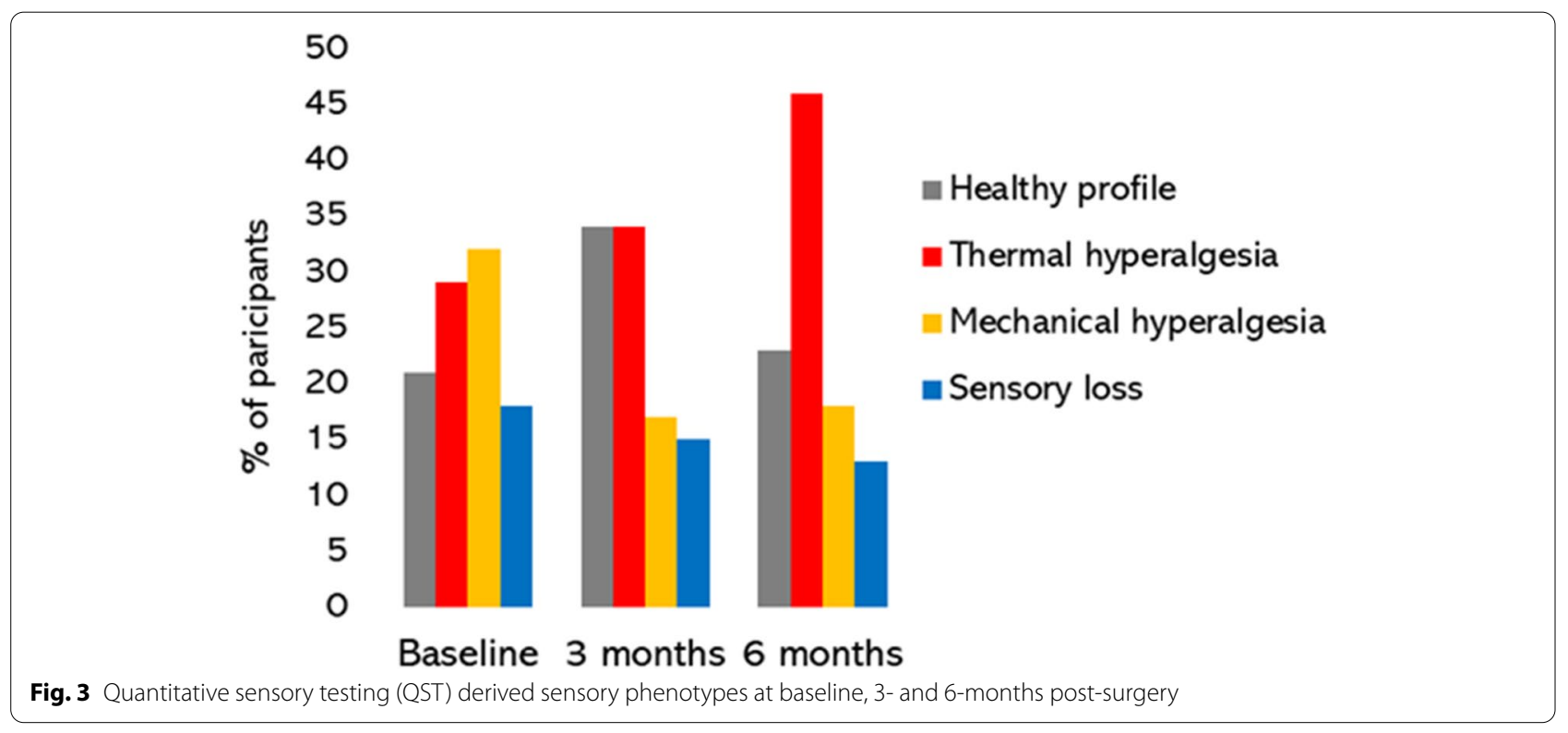




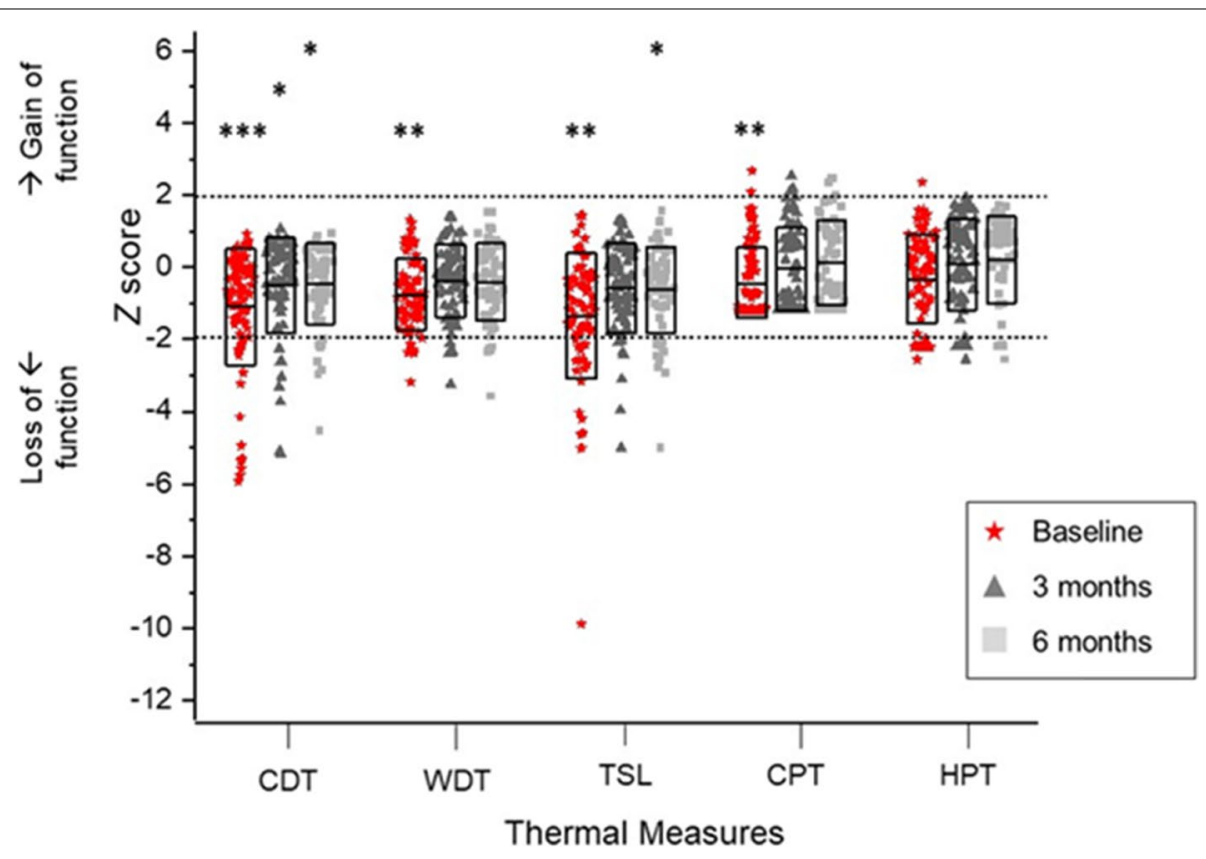

Fig. 4 QST thermal measures comparing CTS participants and control data. Boxes represent the interquartile range, the centre line the median. The black upper dotted line represents $+1.96 z$, the bottom dotted line $-1.96 z$. Scores between the two are interpreted as normal, those above as gain of function and below as loss of function. Significance is denoted as * at the 0.05 probability level; ** at $0.01 ;{ }^{* * *}$ at 0.001 . Cold detection threshold (CDT); cold pain threshold (CPT); heat pain threshold (HPT); thermal sensory limen (TSL); warm detection threshold (WDT)

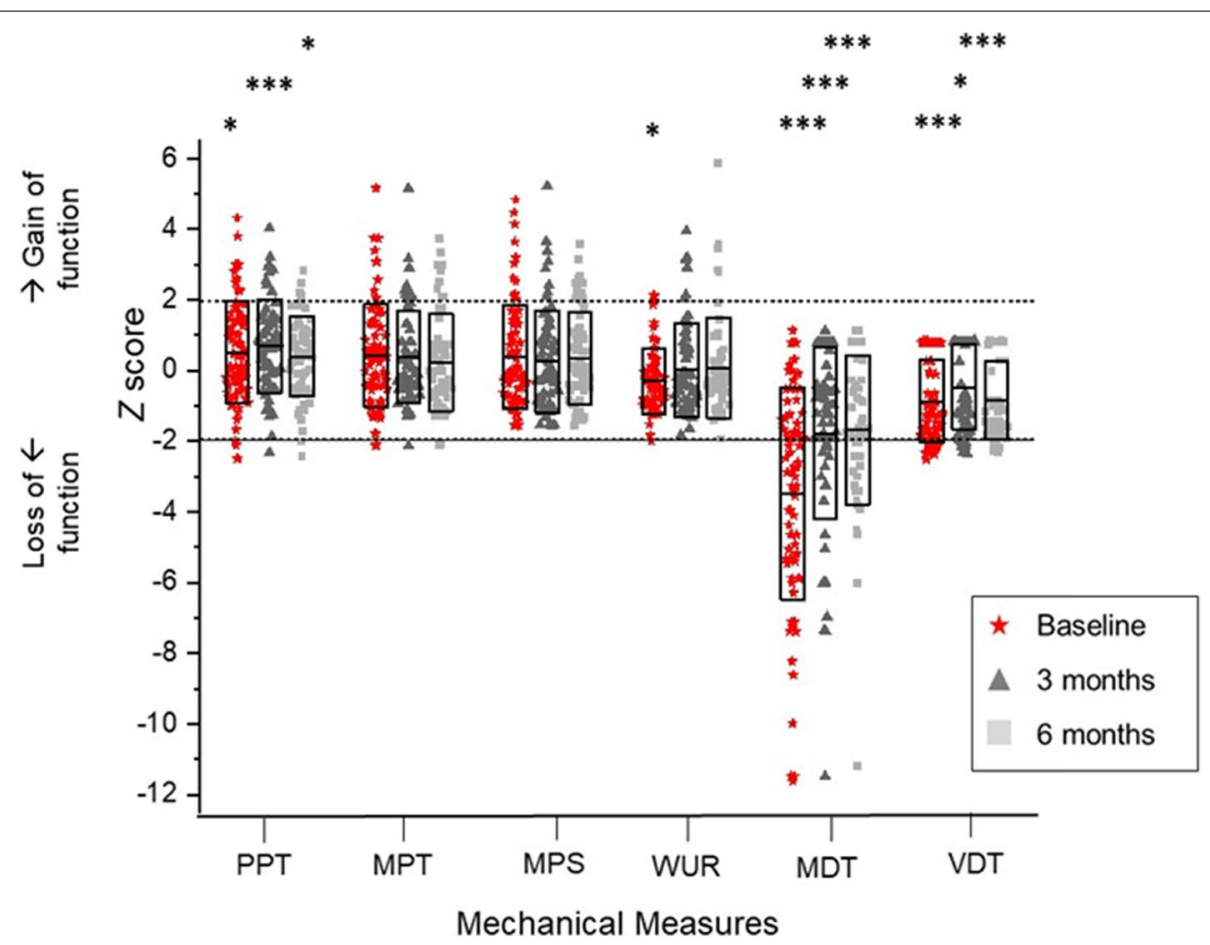

Fig. 5 QST mechanical measures comparing CTS participants and controls. Boxes represent the interquartile range, the centre line the median. The black upper dotted line represents $+1.96 \mathrm{z}$, the bottom dotted line - 1.96z. Scores between the two are interpreted as normal, those above as gain of function and below as loss of function. Significance is denoted as * at the 0.05 probability level; ${ }^{* *}$ at $0.01 ; * * *$ at 0.001 . Mechanical detection threshold (MDT); mechanical pain sensitivity (MPS); mechanical pain threshold (MPT); pressure pain threshold (PPT); vibration detection threshold (VDT); wind-up ration (WUR) 
Table 2 Change in DN4 and incidence of neuropathic pain

\begin{tabular}{llll}
\hline & Baseline $(\boldsymbol{n}=\mathbf{7 6})$ & $\mathbf{3}$ months $(\boldsymbol{n}=\mathbf{6 5})$ & $\mathbf{6}$ months $(\boldsymbol{n}=\mathbf{6 1})$ \\
\hline DN4 mean (sd) & $5.39(2.05)$ & $1.81(1.77)$ & $1.29(1.91)$ \\
Pain Category & & & $25(39)$ \\
$\quad$ Pain free n (\%) & $7(9)$ & $33(51)$ & $29(48)$ \\
Non-neuropathic n (\%) & $11(15)$ & $7(11)$ & $25(41)$ \\
Neuropathic $n(\%)$ & $58(76)$ & $7(12)$ \\
\hline
\end{tabular}

Key: standard deviation (sd)

Table 3 Pain parameters at baseline \& 6 months post-surgery by sensory phenotype

\begin{tabular}{|c|c|c|c|c|c|c|}
\hline \multirow[t]{2}{*}{ Baseline Sensory Phenotype } & \multicolumn{2}{|c|}{ Symptom Severity Score } & \multicolumn{2}{|c|}{ BPI Pain Severity Score } & \multicolumn{2}{|l|}{ NPSI } \\
\hline & baseline & 6 months & baseline & 6 months & baseline & 6 months \\
\hline healthy profile & $2.87(0.8)$ & $1.36(0.6)$ & $3.25(4)$ & $0.75(3.3)$ & $31.5(40.5)$ & $0(9.5)$ \\
\hline thermal hyperalgesia & $3.18(1.3)$ & $1.23(0.4)$ & $5.0(5.3)$ & $0.0(0.4)$ & $29.0(47.0)$ & $2(4.0)$ \\
\hline mechanical hyperalgesia & $3.27(1.2)$ & $1.50(1.6)$ & $3.88(4.7)$ & $0.75(4.5)$ & $32.5(38.5)$ & $7(29.0)$ \\
\hline sensory loss & $3.27(1.8)$ & $1.73(1.2)$ & $5.0(4.2)$ & $0.38(2.6)$ & $39(35.25)$ & $5.5(27.3)$ \\
\hline$p=$ & 0.39 & 0.08 & 0.42 & 0.11 & 0.43 & 0.04 \\
\hline
\end{tabular}

Data reported with median (interquartile range)

Baseline sensory phenotype: healthy profile $n=16,6$ months $n=13$; thermal hyperalgesia $n=22$, baseline $n=20$; mechanical hyperalgesia $n=24,6$ months $n=18$; sensory loss $n=14,6$ months $n=10$. Statistical significance reported for Kruskal-Wallis test

NPSI Neuropathic Pain Symptom Inventory, SSS Symptom Severity Scale score

phenotypic groups were explored prior to and post-surgery. At baseline, scores are lower in those with a healthy sensory phenotype and highest in those with a sensory loss phenotype, however group differences are not significant $(p=0.43)$. While between group differences were statistically significant at 3 months post-surgery $(p=.007)$; differences at 6 months post-surgery were not $(p=0.12)$.

\section{Mood}

The DAPOS scales for depression, anxiety and positive outlook were analysed separately, there is no "total" DAPOS score. No change in depression scores were observed post-surgery for the sample (baseline median 6 [IQR 3]; 6 months median 5 [IQR 2] $(p=.42)$. In contrast, following surgery, a significant decrease in anxiety and increase in positive outlook was observed. Baseline anxiety score (median 4 [IQR 4]) decreased at 3 months (median 3.5 [IQR 2]) $(p=.02)$ and 6 months (median 4 [IQR 2]) $(p=.04)$ while positive outlook scores increased from baseline (median 11 [IQR 5]) to 3 months (median 12 [IQR 5]) and 6 months (median 11 [IQR 5]) $(p \leq .02)$.

\section{Sleep impairment}

Interrupted sleep is a hallmark of CTS and as anticipated, clinically relevant insomnia was prevalent in the sample. At baseline, scores for the Insomnia Severity Index (ISI) indicated 49(64\%) participants had insomnia ranging from subthreshold to severe, with insomnia persisting in 31(46\%) participants at 3 months post-surgery. Change in ISI scores across the 3 assessments was statistically significant; baseline [(median (IQR) 10.0 (11.25)]; 3-months post-surgery 5.5 (11.5), 6 months post-surgery 7 (11.5)] $P<.001$. Differences observed in the severity of sleep impairment, based on baseline sensory phenotype, were not statistically significant $(p>.05)$ (Fig. 7).

\section{Functional interference}

Pain interference and functional impairment was disparate at baseline, with a large proportion of participants reporting moderate to severe dysfunction and interference. (Fig. 8a; 8b). For the sample, Functional Status Scores improved from baseline [mean (standard deviation) $2.64(.85)$ to 3 months $1.96(.89)$ and baseline to 6 months 1.8 (.9) with a large effect size (multivariate partial eta squared $=.443$ ) (Fig. 8a). Consistently, BPI pain interference scale scores improved from baseline [mean (SD) 3.64 (2.28)] to 3 months 1.52 (1.83)] and baseline to 6 months [1.61 (2.19)] with a large effect size (multivariate partial eta squared $=.483$ ) (Fig. $8 \mathrm{~b})$. 


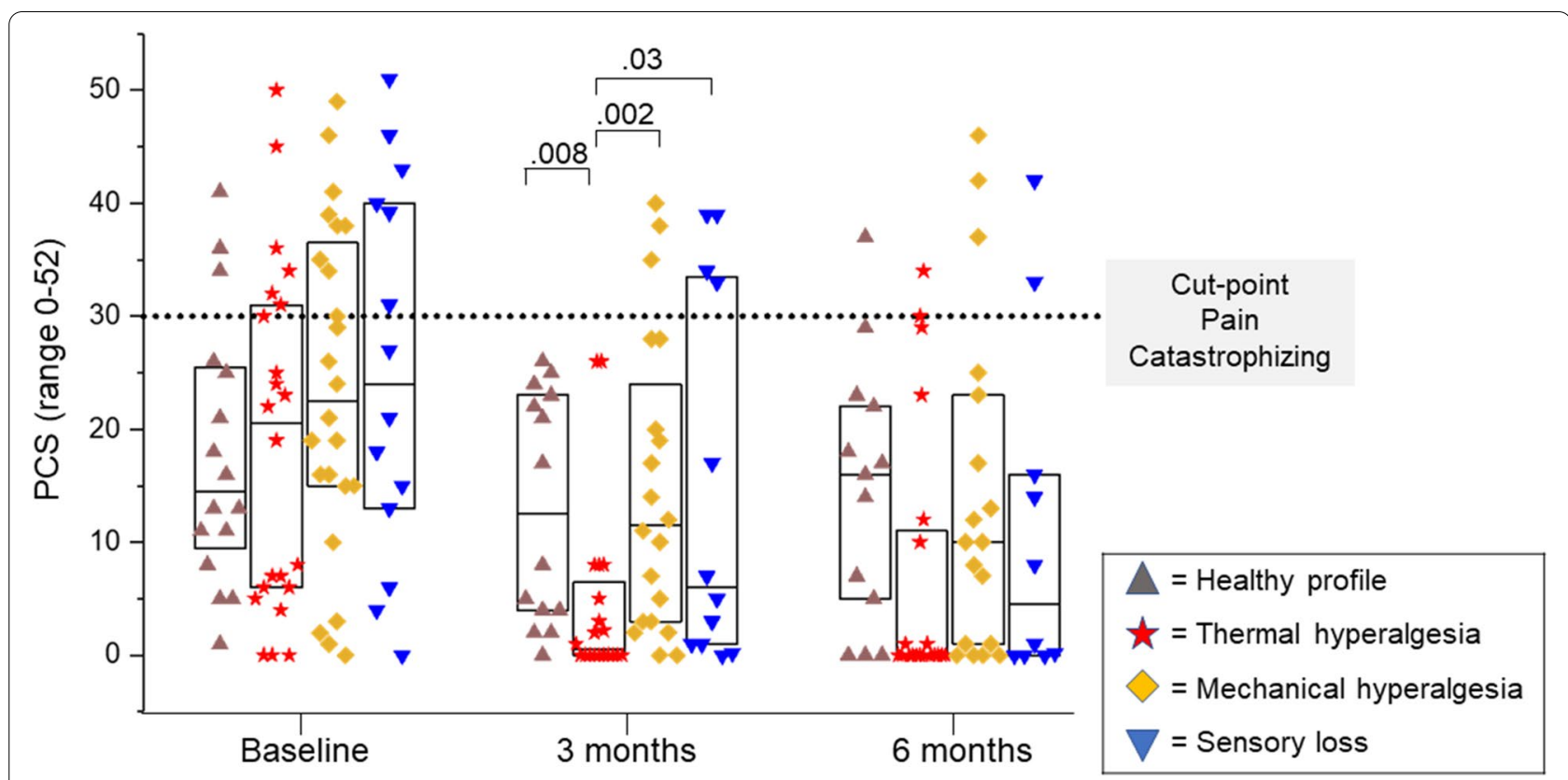

Fig. 6 Pain Catastrophizing Scale (PCS). Differences in total Pain Catastrophizing Scale Score based on baseline phenotype group, at baseline, 3 - and 6 -months post-surgery. Box represents 25 th and 75 th percentiles, centre line the median. Statistically significant differences are reported in bold

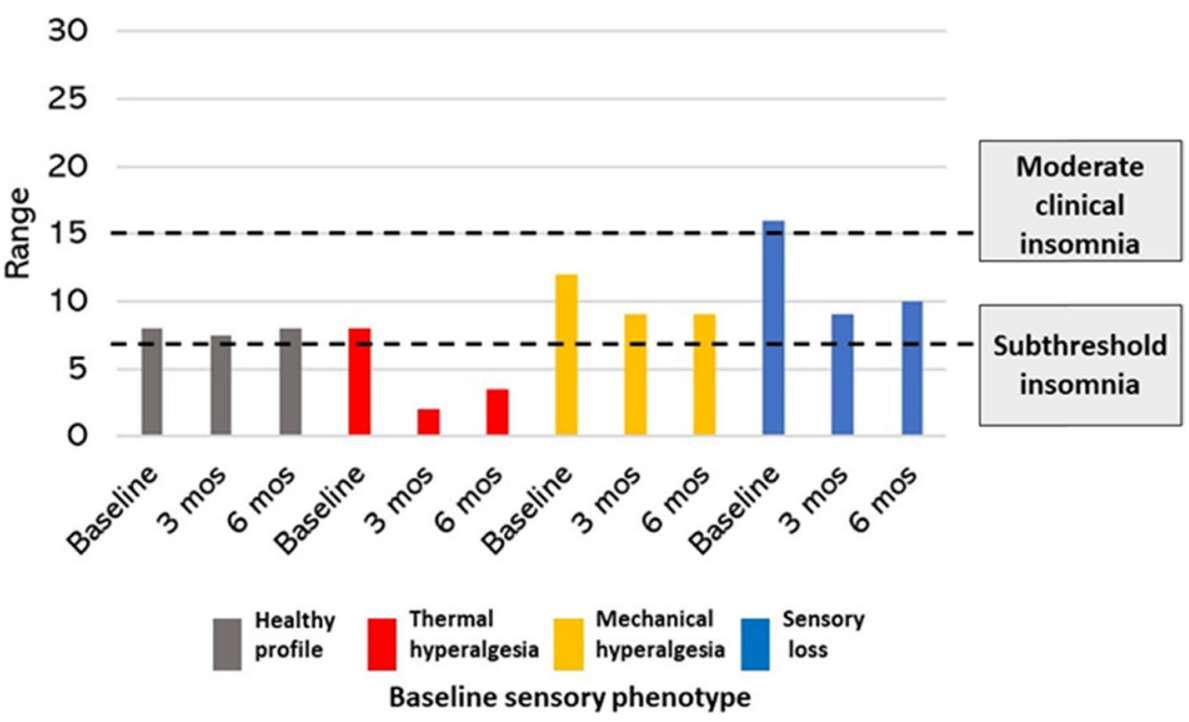

Fig. 7 Median Insomnia Severity Index (ISI) score stratified by baseline sensory phenotype

\section{Sensory phenotype associated burden}

Pre-surgical health status, mood and functional deficit have been identified as potential prognostic factors for the outcome of carpal tunnel surgery. Therefore, we included these measures in an exploration of attendant burden related to QST derived sensory phenotype
(Table 4). At baseline, CTS participants with mechanical hyperalgesia and sensory loss phenotypes have higher comorbidity scores, more severe sleep restriction and worse pain interference and functional impairment however these phenotypic differences did not reach statistical significance. 


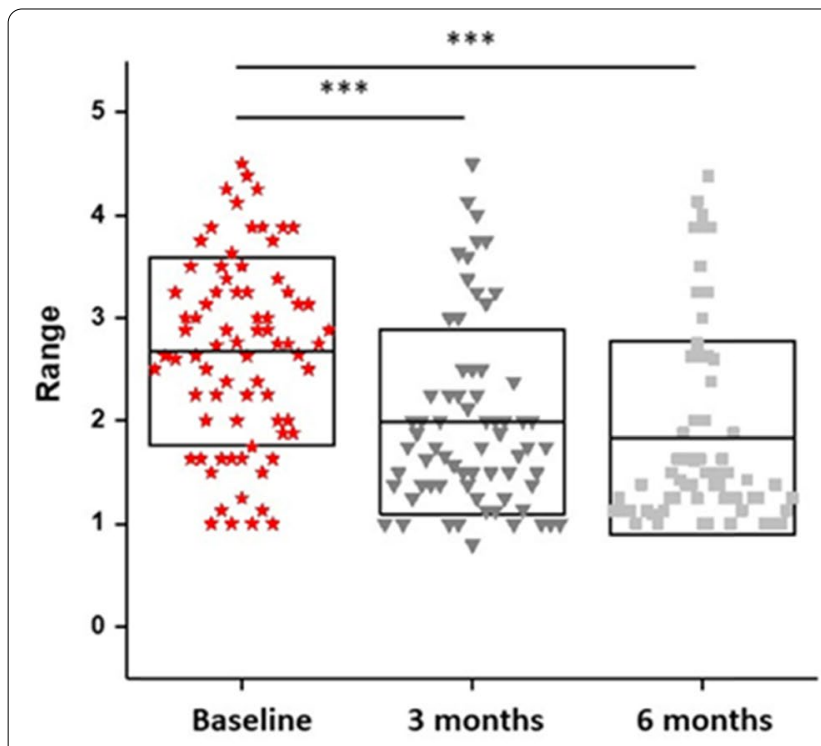

a

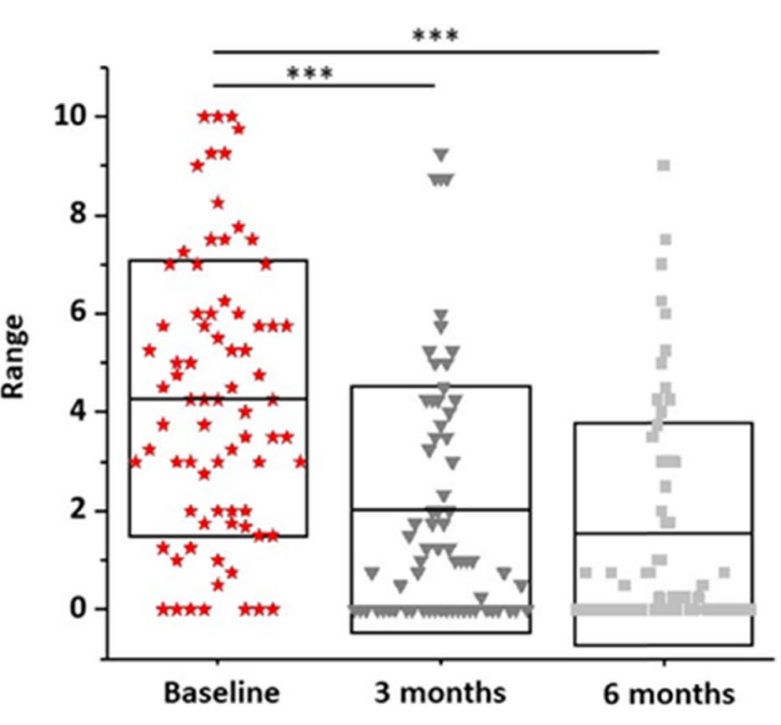

b

Fig. 8 a Functional Status Scores (FSS). b BPI Pain Interference Scale. Change in functional status and pain interference scores across assessments. The grey boxes represent the standard deviation, centre line the mean at baseline, 3- and 6-months post-surgery. Each symbol represents a participant. Significance is denoted as * at the 0.05 probability level; ** at $0.01 ; * *$ at 0.001

Table 4 Comparison of multimodal phenotyping measures between phenotypic groups at baseline

\begin{tabular}{|c|c|c|c|c|c|}
\hline & Healthy Profile & Thermal Hyperalgesia & $\begin{array}{l}\text { Mechanical } \\
\text { Hyperalgesia }\end{array}$ & Sensory loss & $p=$ \\
\hline Comorbidity Score & $3(4)$ & $4.5(7)$ & $5(7)$ & $4(8)$ & .48 \\
\hline Depression & $7(5)$ & $5(2)$ & $5.5(6)$ & $7(4)$ & .27 \\
\hline Anxiety & $4.5(5)$ & $3(4)$ & $5.5(5)$ & $4(5)$ & .58 \\
\hline Insomnia Severity & $8(13)$ & $8(11)$ & $12(11)$ & $16(14)$ & .21 \\
\hline BPI Pain Interference & $2.72(3.57)$ & $3.57(3.61)$ & $3.57(4.22)$ & $5.35(3.72)$ & .15 \\
\hline Functional Status Score & $2.32(1.19)$ & $2.75(1.65)$ & $2.76(1.25)$ & $3.00(1.6)$ & .19 \\
\hline
\end{tabular}

Data reported with median (interquartile range). Statistical significance reported for Kruskal-Wallis test. Baseline $N=76$; healthy profile $n=16$ ( $21 \%$ ); thermal hyperalgesia $n=22$ (29\%); mechanical hyperalgesia $n=24$ (32); sensory loss $n=14$ (18\%)

\section{Surgical outcome}

At 6 months post-surgery, 5 (8\%) of participants reported a poor surgical outcome (worse or unchanged), 59 (92\%) a good outcome (slightly better, much better or completely cured) (Table 5). At 3 months post-surgery 58 (85\%) of participants reported scar pain which ranged from very mild to very severe (Fig. 9). Scar pain severity was diminished at 6 months however $42(65 \%)$ of participants continued to report pain (very mild, 16 (25\%); mild 15 (23\%); moderate $10(16 \%)$ and severe, 1 (1.6\%). At 3 months post-surgery, 50 (74\%) of participants reported some degree of scar interference, this was further reduced at 6 months with $30(47 \%)$ participants reporting some degree of functional interference (Fig. 9).

\section{Candidate prognostic outcome factors}

Observed differences in pain, sleep impairment, psychological factors and function, between sensory phenotypic groups, was not significant. However, because sample size

Table 5 Patient-completed global rating of change at 3 and 6 months

\begin{tabular}{llll}
\hline Rating & $\begin{array}{l}\text { 3 months } \\
\boldsymbol{n}=\mathbf{6 8} \mathbf{n}(\%)\end{array}$ & $\begin{array}{l}\mathbf{6} \text { months } \\
\boldsymbol{n}=\mathbf{6 4} \mathbf{n}(\%)\end{array}$ & \\
\hline Worse & 0 & $2(3 \%)$ & poor outcome $8 \%$ \\
Unchanged & $6(9 \%)$ & $3(5 \%)$ & \\
Slightly better & $11(16 \%)$ & $8(13 \%)$ & good outcome 92\% \\
Much better & $41(69 \%)$ & $33(52 \%)$ & \\
Completely cured & $10(15 \%)$ & $18(28 \%)$ & \\
\hline
\end{tabular}




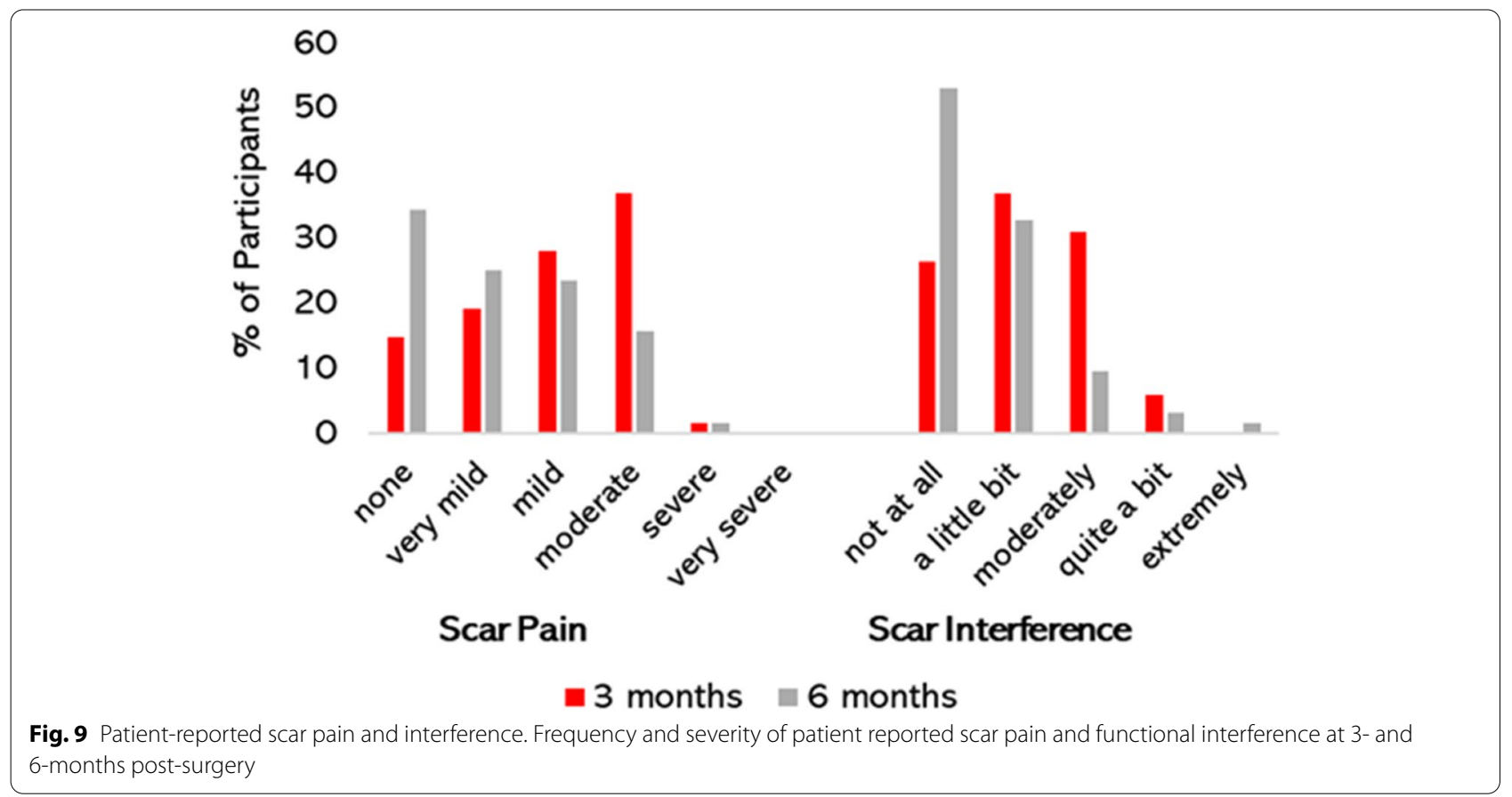

was predicated on one prognostic factor (difference in QST for a CTS cohort and controls), the study may have been under powered to detect between phenotypic group differences. The association of QST derived sensory phenotype, participant demographics and baseline multimodal phenotyping measures including pain parameters, psychological factors, sleep and functional impairment measures with patient global rating of change at 6 months post-surgery were investigated to identify potential factor candidates related to surgical outcome (Table 6). While differences in patient reported outcome between baseline sensory phenotypic groups were observed; a good surgical outcome was reported by $100 \%$ of those with a healthy sensory phenotype, 95\% thermal hyperalgesia, $91 \%$ sensory loss and $85 \%$ mechanical hyperalgesia, these differences were not statistically significant $(p=.51)$. Neither were the participant's age, BMI, Comorbidity Score, duration of symptoms, nerve conduction study measured disease severity or pain parameters associated with surgical outcome $(p>.05)$. However, the association of baseline psychological factors and functional interference with surgical outcome was statistically significant. The Pain Catastrophizing Scale score $(r=-.248, p=.048)$; anxiety subscale of the DAPOS $(r=-.366, P=.003)$ and

Table 6 Correlation of baseline phenotyping parameters with patient reported global rating of change at 6 months

\begin{tabular}{llll}
\hline Patient global rating of change (PGRC) & & & \\
\hline & & $r=$ & Sig. (2-tailed) \\
\hline PAIN PARAMETERS & BPI pain severity score & -.185 & .142 \\
& DN4 & -.227 & .071 \\
& Symptom Severity Score & -.136 & .284 \\
& NPSI & -.180 & .154 \\
PSYCHOLOGICAL MEASURES & Pain Catastrophizing Scale & -.248 & .048 \\
SLEEP & Depression & -.221 & .079 \\
FUNCTION & Anxiety & -.366 & .003 \\
& Insomnia severity index & -.115 & .366 \\
& Functional severity score & -.243 & .053 \\
BPI pain interference scale & $\mathbf{. 3 6 3}$ & .003
\end{tabular}

Correlations interpreted as small $r=.10$ to .29 ; medium $r=.30$ to .49 ; large $r=.50$ to 1.0

BPI Brief Pain Inventory, NPSI Neuropathic Pain Symptom Inventory, sig statistical significance 
BPI Interference scale $(r=-.363, p=.003)$ were inversely correlated with patient reported surgical outcome; greater pain-related worry, greater anxiety and higher levels of functional interference were associated with a poorer surgical outcome.

\section{Discussion}

In patients with chronically painful conditions, the large degree of inter-patient variability in treatment response has rationalized the need to identify measurable phenotypic characteristics of patients that are predictive of treatment outcomes [56]. This longitudinal study adopted such a multimodal phenotyping approach. To identify candidate prognostic factors, we explored the association of sensory phenotype and concomitant pain parameters, psychological factors, sleep restriction and functional impairment with outcome at 6 months post carpal tunnel surgery.

In this sample, a good surgical outcome was reported by $92 \%$ of participants. However, in this pragmatic cohort, a lenient cut-point of 3 (slightly better) was chosen a priori to determine surgical success. If a more stringent cut-point of grade of 4 (much better) was taken, then a good outcome would have been reported by $80 \%$ of participants and is more in keeping with the literature [7]. Our findings demonstrate that greater pain-related worrying, anxiety and functional interference, prior to surgery, are associated with poorer surgical outcome.

\section{Sensory phenotype and surgical outcome}

In patients with peripheral neuropathic pain, the frequency of DFNS QST derived sensory phenotypes, thought to reflect different neurobiological mechanisms, differ between aetiologies [6]. In the current sample, at baseline, approximately $20 \%$ of participants presented with a healthy sensory profile, $30 \%$ with a thermal hyperalgesia phenotype, $30 \%$ with a mechanical hyperalgesia phenotype and approximately $20 \%$ with a sensory loss phenotype. This distribution of phenotypes is comparable to that reported in patients with peripheral nerve injury [6]. Observed differences in surgical outcome, between sensory phenotypic groups, were not significant. However, of clinical importance, while paraesthesiae and numbness are thought to be pathognomonic of carpal tunnel syndrome [2], our findings demonstrate that thermal and mechanical hyperalgesia sensory perturbations are observed as the dominant sensory feature in subgroups of this clinical cohort. Intriguingly, our findings demonstrate significant differences, between sensory phenotypic groups, in pain trajectory. At 6 months postsurgery, significant differences are observed for the Neuropathic Pain Severity Index whereby participants with mechanical hyperalgesia and sensory loss phenotypes demonstrate greater persisting neuropathic pain scores, perhaps supporting the hypothesis that sensory phenotype reflects different neurobiological mechanisms.

In this sample, prior to decompression surgery, loss of thermal detection (small fibre) and/or mechanical detection (large fibre) was more prevalent than gain to thermal or mechanical stimuli. Post-surgery, significant improvement is observed in large fibre function (vibration and mechanical detection), however at 6 months sensory function remains impaired compared to healthy controls, demonstrating recovering but persistent dysfunction. In contrast, a greater degree of small fibre function recovery is observed post-surgery. Cold detection threshold and thermal sensory limen improve significantly but remain reduced at 6 months, whereas warm detection threshold and cold pain threshold normalise at 3 months. These findings are consistent with the work of Baskozos et al. [75] who demonstrate with quantitative sensory testing, histologically and electrodiagnostically, that while improvement in large and small fibre encoded modalities is observed following median nerve decompression, recovery remains incomplete at 6 months. It is unclear, beyond 6 months, if large and small median nerve fibre function recovers further.

\section{Exploring pain in carpal tunnel syndrome}

Findings for pain and symptom severity measures demonstrate that for patients with CTS, pain intensity, frequency and quality are heterogeneous experiences. At baseline $91 \%$ of participants reported pain of some severity (mean [standard deviation] pain severity score 4.17 [2.73]) however, of interest, pain parameters at baseline were not associated with patient rated surgical outcome. A significant improvement (reduction) for all pain parameters (DN4, NPSI, Symptom Severity Score and BPI pain severity score) was observed at 3 and 6 months post-surgery, consistent with findings in other carpal tunnel surgery cohorts $[66,76]$, however this does not imply pain resolution for all patients, as clearly illustrated.

In CTS, the mechanisms driving pain are not clearly elucidated and may change over the course of the disease. Theories based on the pathophysiology of nerve compression implicate ischemia secondary to raised intraneurial pressure, fibrosis and subsequent traction on a nerve that has been rendered immobile or possibly localised inflammatory processes beneath the transverse carpal ligament [26, 27]. It is intriguing that in CTS, patient-reported pain and symptom severity do not correlate with the severity of electrophysiologically assessed nerve conduction delay [14, 77, 78], in other words, more severe compression does not drive more severe pain. 
Determining whether pain that is symptomatic of carpal tunnel syndrome is neuropathic in nature is important as this may have implications for treatment. Equally, it is important to understand the nature of persistent post-surgical pain in this group of patients for the same reason. Estimates of neuropathic pain in patients with carpal tunnel syndrome have ranged from 48 to $80 \%$ based on the tools used for diagnosis and sample characteristics [24, 77, 79]. In the current study, a stringent two-stage triage was used to define pain as neuropathic, or not [34] and $76 \%$ of participants were categorised as having neuropathic pain prior to surgery. Confirming the persistence of impaired median nerve somatosensory function, i.e., the persistence of a nerve lesion, post-operatively is essential in the diagnosis of post-operative neuropathic pain. In the current sample, at 6 months post-surgery, the incidence of neuropathic pain was found to be $12 \%$, with $48 \%$ reporting pain that was non-neuropathic in nature and $40 \%$ reporting they were pain-free.

For many patients with CTS, pain control prior to surgery is not adequate. Furthermore, for a proportion of patients who undergo carpal tunnel decompression surgery, pain is not resolved. This unmet need has spurred a growing interest in the efficacy of vitamins and nutraceuticals for the reduction of pain and symptom severity in this patient population. Of particular interest, alphalipoic acid (ALA) is reported to exert an antioxidative, anti-inflammatory effect on peripheral nerves, thereby reducing sensory symptoms associated with peripheral neuropathy [80]. In patients with CTS, the administration of oral ALA both prior to and/or after surgery has been investigated in several blinded, randomised controlled trials with results suggesting some degree of efficacy in symptom and pain reduction [81-84]. While ALA appears a promising nutraceutical for the reduction of sensory symptoms in patients with CTS as well as improvement in carpal tunnel surgical outcomes, this evidence must be interpreted with some caution. Heterogeneity in study design including variability in ALA dose and frequency, disparity in the CTS symptom parameters of interest and the outcome evaluation of such and queries as to the adequacy of study sample sizes $[82,83]$ to detect between-groups differences suggest that further evidence of efficacy may be required.

\section{Pain-related worrying}

Pain catastrophizing, or exaggerated, persistent thoughts related to painful experiences [67] has emerged as one of the most important psychological predictors of pain, distress, and disability [85]. However, a recent content analysis of pain catastrophizing self-report measures has raised questions as to the validity of assessing pain catastrophizing via self-report and suggests the construct being measured is more appropriately described as 'pain-related worrying' [69]. Simultaneously, a large international patient-researcher collaboration has rallied for a replacement term for pain catastrophizing in light of patient concerns that the term is pejorative, stigmatising and poses a barrier to care [86]. Therefore, the term pain-related worry has been used in the current work to describe the construct in question.

At baseline 33\% of study participants were categorized as being high in pain-related worry based on their Pain Catastrophizing Scale (PCS) score. There was a significant decrease in scores up to 6 months, at which time $13 \%$ continued to report a high degree of pain-related worry. There is debate as to the nature of pain catastrophising as a construct, whether pain catastrophising is representative of a stable state or a situational trait $[85,87]$. Our findings demonstrate a significant reduction in PCS scores $(p<.001)$ with a parallel reduction or improvement in pain severity scores $(<.001)$ from baseline to 6 months post-surgery, suggesting that when pain is effectively treated, catastrophic thought or pain-related worry diminishes.

In this sample, greater pain-related worry, prior to surgery, was found to be associated with poorer surgical outcome. This finding is supported by a recent prospective, observational study in 417 patients undergoing carpal tunnel decompression surgery; Mosegaard et al. [88] reported a statistically significant effect of preoperative Pain Catastrophizing Scale score on patient reported satisfaction.

\section{Anxiety}

For three quarters of a century, preoperative anxiety has been recognized as a potentially modifiable risk factor for post-surgical complications $[89,90]$. It is estimated that between 25 and $80 \%$ of patients admitted to hospital for surgery experience preoperative anxiety [91] and recognised that preoperative anxiety can adversely affect patient recovery [92]. In the current study, anxiety, as investigated with the DAPOS questionnaire, decreased significantly across all time points. Importantly, higher baseline anxiety scores were associated with poorer surgical outcome. This is consistent with findings from a large, multicentre cohort study of patients undergoing carpal tunnel surgery; a significant relationship was identified between anxiety and surgical outcome in this surgical cohort [23].

\section{Sleep disturbance}

Surprisingly, in this sample of patients, Insomnia Severity Index (ISI) scores were not associated with patient-rated outcome. At baseline, ISI scores for $64 \%$ of participants suggested insomnia ranging from subthreshold to severe in intensity while at 6 months the frequency of insomnia 
was decreased to $46 \%$ of participants. In CTS, sleep interruption is a hallmark of the condition and is reported as a primary driver for patients to seek surgery. It is clear from the literature that the bidirectional relationship of sleep disturbance and pain is complex. A recent systematic review and meta-analysis in clinical populations reported that impaired sleep quality and quantity are associated with an increased risk of developing a chronic pain condition, small elevations in inflammatory markers and worse patient reported function [93]. There is also evidence from experimental studies that chronic insufficient sleep can alter pain modulation processes and induce sensitization, thereby increasing vulnerability to chronic pain [94, 95]. It is not clear, however, how or if these pain sensitisation processes normalise when sleep improves or normalises. It is possible that in the current cohort sleep impairment improved post-surgery but not of a sufficiency or length of time to impact on pain sensitivity, function and secondarily on patient-rated surgical outcome. It is also possible that sleep impairment was overestimated by participants both pre and post-surgery. Such a disparity between objective and subjective sleep measures is commonly reported in the literature [96] and is known to confound the evaluation of sleep and related impairment.

\section{Functional impairment}

Reportedly, greater functional impairment prior to surgery, as evaluated with the Functional Status Scale (FSS) of the Boston Carpal Tunnel, is associated with poorer long-term outcome following carpal tunnel surgery [22]. In the present cohort of patients, the association of function as assessed with the FSS approached but did not reach significance $(p=.053)$. However, a significant association was identified between baseline pain functional interference scores and patient reported global rating of change at 6 months post-surgery. The FSS comprises eight hand function specific items, including writing, fastening buttons and opening jars. In contrast, the pain interference scale of the Brief Pain Inventory (BPI) explores the degree to which pain interferes with seven areas of function, including general activity, mood, walking, work, interpersonal relationships, sleep and enjoyment of life [62]. The two measures therefore may be evaluating different constructs and considering function at different levels, with the FSS evaluating function at the impairment level and the BPI interference scale at the participation level.

\section{Scar pain and interference}

At 6 months post- surgery $65 \%$ of CTS participants continued to report some degree of surgical scar pain and $47 \%$ reported scar interference. This finding is in keeping with the literature, scar pain and interference are reported to persist in some patients at up to 2 years post-surgery [97-99]. Despite evidence of the high incidence of problematic surgical scars following carpal tunnel surgery, patients are not routinely counselled prior to surgery as to this possibility of this adverse event and this is an important area for practice improvement.

\section{Study limitations}

Study sample size was determined to enable the detection of a difference in somatosensory function (via quantitative sensory testing) between patients undergoing carpal tunnel surgery and healthy controls. Therefore, the study may have been underpowered to detect between-group differences in other phenotyping measures.

It was anticipated that up to $25 \%$ of participants enrolled in this study would report a poor surgical outcome, enabling the use of multivariate analysis of variance or regression analysis to robustly identify candidate prognostic factors. However, as only a small proportion of patients reported a poor outcome this was precluded; correlation was used to identify the association of phenotyping measures and surgical outcome.

At both study sites, surgery was performed by multiple surgeons. While the operating surgeon was not included as an outcome variable, patient-reported surgical outcome was consistent with that reported in the literature.

All patients scheduled for carpal tunnel surgery were invited to participate, however it is impossible to control for participant-selection bias. In clinical studies of this nature, the clinician-patient interaction cannot be ruled out and may influence or bias the patients' perception or judgment of outcome. To reduce the likelihood of assessor bias the investigator (DLK) was blinded to patient reported results of surgical outcome until patients completed the trial. The completed measures were placed in pre-labeled, coded envelopes by the study participants and secured in the written case report form. As the baseline and post-surgical measures were completed by one investigator in this study, one cannot rule out the risk of investigator bias.

Study findings may not be generalizable to other care pathways, for example in settings where there is minimal waiting time from patient presentation to surgery.

\section{Conclusions}

This study explored the association of QST derived sensory phenotype and associated comorbid burden with the outcome of carpal tunnel surgery with the aim of identifying candidate prognostic outcome factors for future investigation. Our findings demonstrate that patients with CTS present with significant heterogeneity in somatosensory function; while post-surgical recovery is observed, dysfunction persists in both small and large fibre function at 6 months. Differences between sensory phenotypic groups in pain parameters, pain catastrophising, mood, insomnia 
and functional impairment were observed, suggesting greater burden in patients with a mechanical hyperalgesia or sensory loss phenotype, however these differences did not reach statistical significance in this sample size. Similarly, phenotypic group differences in surgical outcome were observed but did not reach statistical significance. In patients with CTS, pain-related worry is prevalent but improves significantly post-surgery with a concomitant reduction in pain. Sleep impairment, assumed secondary to symptom severity, persists in a large proportion of patients post-surgery, despite symptom reduction. Greater painrelated worrying, anxiety and pain interference prior to surgery, are associated with poorer surgical outcome. These candidate prognostic outcome factors require further investigation in prognostic factor modelling studies.

\section{Clinical implications}

- There is a growing body of evidence that pain-related worry and anxiety are associated with the outcome of carpal tunnel surgery. At present, pre-surgical clinical assessment does not routinely include the evaluation of known psychological risk factors. Where high levels of anxiety are identified, cognitive behavioural therapy (CBT) has been shown to be effective [100] and warrants consideration for implementation in surgery "prehabilitation" programmes. In addition, patient codesign of surgical care pathways might be explored, to ensure that pathways are patient-centred to the greatest degree possible.

- Prior to carpal tunnel surgery, patients should be informed as to the possibility of prolonged or persistent scar discomfort and interference. Scar outcomes have important implications particularly so for those returning to manual work.

- Consistent with previous reports [101], patients with carpal tunnel syndrome do not present with dynamic mechanical allodynia, neither prior to nor following decompression surgery. Allodynia is frequently observed in patients with complex regional pain syndrome (CRPS) [28], a severe, debilitating chronic pain condition which is known to occur, albeit rarely, as a potential severe complication of carpal tunnel decompression surgery [102]. Allodynia is not pathognomonic of CRPS, however where detected in patients with CTS or following carpal tunnel surgery investigation to rule out CRPS is warranted.

\footnotetext{
Abbreviations

ANOVA: Analysis of variance; BCTQ: Boston Carpal Tunnel Questionnaire; BPI: Brief Pain Inventory; BPI-SF: Brief Pain Inventory Short Form; CTR: Carpal tunnel release; CTS: Carpal tunnel syndrome; CBT: Cognitive behavioural therapy; CDT: COLD detection threshold; CPT: Cold pain threshold; CRPS: Complex regional pain syndrome; DAPOS: Depression, Anxiety and Positive Outlook Scale; DN4: Douleur Neuropathique 4 questions; FSS: Functional Status Scale;
}

DFNS: German Research Network on Neuropathic Pain; HPT: Heat pain threshold; IMMPACT: Initiative on Methods, Measurement, and Pain Assessment in Clinical Trials; ISI: Insomnia Severity Index; MDT: Mechanical detection threshold; MPS: Mechanical pain sensitivity; MPT : Mechanical pain threshold; NHS: National Health Service; NCS: Nerve conduction studies; NPSI: Neuropathic Pain Symptom Inventory; PCS: Pain Catastrophizing Scale; PSS: Pain Severity Score; PGRC: Patient-reported global rating of change; PPT: Pressure pain threshold; QST: Quantitative sensory testing; SSS: Symptom Severity Scale; TSL: Thermal sensory limen; VDT : Vibration detection threshold; WDT: Warm detection threshold; WUR: Wind-up ration.

\section{Acknowledgements}

The authors would like to thank patient collaborators Ms. Siobhan Paull and Mrs. Awatef Taylor for their invaluable assistance in reviewing and editing study documents and piloting study procedures and measures. We additionally wish to thank Mr. Shehan Hettiaratchy and Miss Effie Katsarma for their assistance with recruitment and to thank all the patients who gave of their time to participate in this study.

\section{Disclosures}

This report is independent research and the views expressed in this publication are those of the authors and not necessarily those of the NHS, the National Institute for Health Research, or the Department of Health.

\section{Authors' contributions}

DK, CMA and ASCR designed the study; DK, DR, LL, CMA and ASCR contributed to the study concept and interpretation; DLK completed all participant testing; DK, DR and JV performed statistical analysis; all authors contributed to drafting and revising the manuscript. The author(s) read and approved the final manuscript.

\section{Funding}

DL Kennedy was funded by the National Institute for Health Research and Health Education England Clinical Doctoral Research Fellowship (CDRF 2013-04-009). Infrastructure support for this research was provided by the NIHR Imperial Biomedical Research Centre (BRC). D. Ridout received financial support from the NIHR and HEE for her contribution to this work.

\section{Availability of data and materials}

Healthy volunteer quantitative sensory testing data is available at https://doi. org/10.6084/m9.figshare.12860066.v2. Additional datasets used and/or analysed during the current study are available from the corresponding author on reasonable request.

\section{Declarations}

\section{Ethics approval and consent to participate}

Ethical approval was granted by the Camberwell St Giles National Research Ethics Committee (14/LO/1436) on 29 August 2014. Participants provided informed, written consent for participation.

\section{Consent for publication}

Participants provided informed, written consent for publication.

\section{Competing interests}

A.S.C. Rice: IASP Council Member and Chair 18th World Congress on Pain Scientific Programme Committee; A.S.C.R undertakes consultancy and advisory board work for Imperial College Consultants - in the past 24 months this has included remunerated work for Abide, Pharmanovo, Lateral, Novartis, Pharmaleads, Mundipharma, Orion, Asahi Kasei, Toray, and Theranexis; ASCR was the owner of share options in Spinifex Pharmaceuticals, from which personal benefit was accrued on the acquisition of Spinifex by Novartis in July 2015 and from which payments continued until 2019. ASCR is named as an inventor on the following patents: (1) A.S.C. Rice, S. Vandevoorde and D.M. Lambert Methods using $\mathrm{N}$-(2-propenyl) hexadecanamide and related amides to relieve pain. WO 2005/079771. (2) Okuse K. et al. Methods of treating pain by inhibition of vgf activity EP13702262.0/WO2013 110945. Jan Vollert: personal fees from Vertex Pharmaceuticals, personal fees from Embody Orthopaedic, outside the submitted work. The remaining authors have no conflicts of interest to declare. 


\begin{abstract}
Author details
${ }^{1}$ Pain Research, Department of Surgery and Cancer, Faculty of Medicine, Chelsea \& Westminster Hospital Campus, Imperial College London, 369 Fulham Rd, London SW10 9NH, UK. ${ }^{2}$ Therapies Department, Imperial College Healthcare NHS Trust, London, UK. ${ }^{3}$ Population, Policy and Practice Programme, University College London Great Ormond St Institute of Child Health, London, UK. ${ }^{4}$ Department of Plastic and Reconstructive Surgery, Imperial College Healthcare NHS Trust, London, UK. ${ }^{5}$ Division of Neurological Pain Research and Therapy, Department of Neurology, University Hospital of Schleswig-Holstein, Campus Kiel, Germany. ${ }^{6}$ Department of Anaesthesiology, Intensive Care and Pain Medicine, University Hospital Muenster, Muenster, Germany. ${ }^{7}$ Neurophysiology, Mannheim Center of Translational Neuroscience (MCTN), Medical Faculty Mannheim, Heidelberg University, Heidelberg, Germany. ${ }^{8}$ MSk Lab, Department of Surgery and Cancer, Imperial College London, London, UK.
\end{abstract}

Received: 31 August 2021 Accepted: 25 October 2021 Published online: 17 November 2021

\section{References}

1. Olney RK. Carpal tunnel syndrome: complex issues with a "simple" condition. Neurology. 2001;56(11):1431-2.

2. Padua L, Coraci D, Erra C, Pazzaglia C, Paolasso I, Loreti C, et al. Carpal tunnel syndrome: clinical features, diagnosis, and management. Lancet Neurol. 2016;15(12):1273-84.

3. Huisstede BM, Randsdorp MS, Coert JH, Glerum S, van Middelkoop M, Koes BW. Carpal tunnel syndrome. Part II: effectiveness of surgical treatments--a systematic review. Arch Phys Med Rehabil. 2010;91(7):1005-24

4. Shi $Q$, MacDermid JC. Is surgical intervention more effective than nonsurgical treatment for carpal tunnel syndrome? A systematic review. J Orthop Surg Res. 2011;6:17.

5. Gerritsen AA, de Vet HC, Scholten RJ, Bertelsmann FW, de Krom MC, Bouter LM. Splinting vs surgery in the treatment of carpal tunnel syndrome: a randomized controlled trial. JAMA. 2002;288(10):1245-51.

6. Verdugo RJ, Salinas RA, Castillo JL, Cea JG. Surgical versus non-surgical treatment for carpal tunnel syndrome. Cochrane Database Syst Rev. 2008;4:CD001552

7. Bland JD. Treatment of carpal tunnel syndrome. Muscle Nerve. 2007;36(2):167-71.

8. Louie D, Earp B, Blazar P. Long-term outcomes of carpal tunnel release: a critical review of the literature. Hand. 2012;7(3):242-6.

9. Banugo P, Amoako D. Prehabilitation. BJA Education. 2017;17(12):401-5.

10. Amadio PC, Silverstein MD, Ilstrup DM, Schleck CD, Jensen LM. Outcome assessment for carpal tunnel surgery: the relative responsiveness of generic, arthritis-specific, disease-specific, and physical examination measures. J Hand Surg. 1996;21(3):338-46.

11. Glowacki KA, Breen CJ, Sachar K, Weiss AP. Electrodiagnostic testing and carpal tunnel release outcome. J Hand Surg. 1996;21(1):117-21.

12. Concannon MJ, Gainor B, Petroski GF, Puckett CL. The predictive value of electrodiagnostic studies in carpal tunnel syndrome. Plast Reconstr Surg. 1997;100(6):1452-8.

13. Bland JD. Do nerve conduction studies predict the outcome of carpal tunnel decompression? Muscle Nerve. 2001;24(7):935-40.

14. Longstaff $L$, Milner RH, O'Sullivan S, Fawcett P. Carpal tunnel syndrome: the correlation between outcome, symptoms and nerve conduction study findings. J Hand Surg. 2001;26(5):475-80.

15. Dennerlein JT, Soumekh FS, Fossel AH, Amick BC 3rd, Keller RB, Katz JN. Longer distal motor latency predicts better outcomes of carpal tunnel release. J Occup Environ Med. 2002:44(2):176-83.

16. Schrijver HM, Gerritsen AA, Strijers RL, Uitdehaag BM, Scholten RJ, de Vet $\mathrm{HC}$, et al. Correlating nerve conduction studies and clinical outcome measures on carpal tunnel syndrome: lessons from a randomized controlled trial. J Clin Neurophysiol. 2005;22(3):216-21.

17. Jarvik JG, Comstock BA, Heagerty PJ, Haynor DR, Fulton-Kehoe D, Kliot $M$, et al. Magnetic resonance imaging compared with electrodiagnostic studies in patients with suspected carpal tunnel syndrome: predicting symptoms, function, and surgical benefit at 1 year. J Neurosurg. 2008:108(3):541-50
18. Naranjo A, Ojeda S, Arana V, Baeta P, Fernandez-Palacios J, Garcia-Duque $O$, et al. Usefulness of clinical findings, nerve conduction studies and ultrasonography to predict response to surgical release in idiopathic carpal tunnel syndrome. Clin Exp Rheumatol. 2009;27(5):786-93.

19. Turner A, Kimble F, Gulyas K, Ball J. Can the outcome of open carpal tunnel release be predicted?: a review of the literature. ANZ J Surg. 2010;80(1-2):50-4.

20. Galasso O, Mariconda M, Donato G, Di Mizio G, Padua L, Brando A, et al. Histopathological, clinical, and electrophysiological features influencing postoperative outcomes in carpal tunnel syndrome. J Orthop Res. 2011;29(8):1298-304

21. Hemingway H, Croft P, Perel P, Hayden JA, Abrams K, Timmis A, et al. Prognosis research strategy (PROGRESS) 1: a framework for researching clinical outcomes. BMJ. 2013:346:e5595.

22. De Kleermaeker F, Meulstee J, Bartels R, Verhagen WIM. Long-term outcome after carpal tunnel release and identification of prognostic factors. Acta Neurochir. 2019;161(4):663-71.

23. Jerosch-Herold C, Shepstone L, Houghton J, Wilson ECF, Blake J. Prognostic factors for response to treatment by corticosteroid injection or surgery in carpal tunnel syndrome (palms study): a prospective multicenter cohort study. Muscle Nerve. 2019;60(1):32-40.

24. Matesanz L, Hausheer AC, Baskozos G, Bennett DL, Schmid AB. Somatosensory and psychological phenotypes associated with neuropathic pain in entrapment neuropathy. Pain. 2020;162(4):1211-20.

25. Kennedy DL, Vollert J, Ridout D, Alexander CM, Rice AS. The responsiveness of quantitative sensory testing-derived sensory phenotype to disease-modifying intervention in patients with entrapment neuropathy: a longitudinal study. Pain. 2021. https://doi.org/10.1097/j.pain. 0000000000002277 . Epub ahead of print

26. Mackinnon SE. Pathophysiology of nerve compression. Hand Clin. 2002:18(2):231-41.

27. Bland JD. Carpal tunnel syndrome. Curr Opin Neurol. 2005;18(5):581-5.

28. Maier C, Baron R, Tolle TR, Binder A, Birbaumer N, Birklein F, et al. Quantitative sensory testing in the German research network on neuropathic pain (DFNS): somatosensory abnormalities in 1236 patients with different neuropathic pain syndromes. Pain. 2010;150(3):439-50.

29. von Hehn CA, Baron R, Woolf CJ. Deconstructing the neuropathic pain phenotype to reveal neural mechanisms. Neuron. 2012;73(4):638-52.

30. Arendt-Nielsen L, Yarnitsky D. Experimental and clinical applications of quantitative sensory testing applied to skin, muscles and viscera. J Pain. 2009;10(6):556-72.

31. Campbell JN, Meyer RA. Mechanisms of neuropathic pain. Neuron. 2006;52(1):77-92

32. Fields HL, Rowbotham M, Baron R. Postherpetic neuralgia: irritable nociceptors and deafferentation. Neurobiol Dis. 1998;5(4):209-27.

33. Max MB. Towards physiologically based treatment of patients with neuropathic pain. Pain. 1990;42(2):131-7.

34. Jensen TS, Baron R, Haanpaa M, Kalso E, Loeser JD, Rice AS, et al. A new definition of neuropathic pain. Pain. 2011:152(10):2204-5.

35. Merskey H, Bogduk N. Part III: Pain Terms, A Current List with Definitions and Notes on Usage. Classification of Chronic Pain, Second Edition, IASP Task Force on Taxonomy. Seattle: IASP Press; 1994. p. 209-14. https://www.iasp-pain.org/Taxonomy?navltemNumber $=576$.

36. Blyth FM. Global burden of neuropathic pain. Pain. 2018;159(3):614-7.

37. WHO. Towards a common language for functioning, disability and health: ICF The International Classification of Functioning, Disability and Health. Geneva; 2002. https://www.who.int/classifications/icf/icfbe ginnersguide.pdf.

38. Haanpää M, Attal N, Backonja M, Baron R, Bennett M, Bouhassira D, et al. NeuPSIG guidelines on neuropathic pain assessment. Pain 2011;152:14-27.

39. Turk DC, Dworkin RH, Allen RR, Bellamy N, Brandenburg N, Carr DB, et al. Core outcome domains for chronic pain clinical trials: IMMPACT recommendations. Pain. 2003:106(3):337-45.

40. Turk DC, Dworkin RH, Revicki D, Harding G, Burke LB, Cella D, et al. Identifying important outcome domains for chronic pain clinical trials: an IMMPACT survey of people with pain. Pain. 2008;137(2):276-85.

41. Tampin B, Vollert J, Schmid AB. Sensory profiles are comparable in patients with distal and proximal entrapment neuropathies, while the pain experience differs. Curr Med Res Opin. 2018;34(11):1-8. 
42. Rolke R, Baron R, Maier C, Tolle TR, Treede RD, Beyer A, et al. Quantitative sensory testing in the German research network on neuropathic pain (DFNS): standardized protocol and reference values. Pain. 2006;123(3):231-43

43. Vollert J, Maier C, Attal N, Bennett DLH, Bouhassira D, Enax-Krumova EK, et al. Stratifying patients with peripheral neuropathic pain based on sensory profiles: algorithm and sample size recommendations. Pain. 2017;158(8):1446-55.

44. Magerl W, Krumova EK, Baron R, Tolle T, Treede RD, Maier C. Reference data for quantitative sensory testing (QST): refined stratification for age and a novel method for statistical comparison of group data. Pain. 2010;151(3):598-605.

45. Zanette G, Cacciatori C, Tamburin S. Central sensitization in carpal tunnel syndrome with extraterritorial spread of sensory symptoms. Pain. 2010;148(2):227-36

46. Kennedy DL, Ridout D, Alexander CM, Rlce ASC. Healthy Volunteer Quantitative Sensory Testing Stud 2020 [cited 2021]. doi: https://doi. org/10.6084/m9.figshare.12860066.v2.

47. Goldsmith R, Wright C, Bell SF, Rushton A. Cold hyperalgesia as a prognostic factor in whiplash associated disorders: a systematic review. Man Ther. 2012;17(5):402-10.

48. Muller M, Butikofer L, Andersen OK, Heini P, Arendt-Nielsen L, Juni $P$, et al. Cold pain hypersensitivity predicts trajectories of pain and disability after low back surgery: a prospective cohort study. Pain. 2021;162(1):184-94.

49. Bland JD, Rudolfer SM. Ultrasound imaging of the median nerve as a prognostic factor for carpal tunnel decompression. Muscle Nerve. 2014;49(5):741-4

50. Jerosch-Herold C, Shepstone L, Wilson EC, Dyer T, Blake J. Clinical course, costs and predictive factors for response to treatment in carpal tunnel syndrome: the PALMS study protocol. BMC Musculoskelet Disord. 2014;15:35.

51. Levine DW, Simmons BP, Koris MJ, Daltroy LH, Hohl GG, Fossel AH, et al. A self-administered questionnaire for the assessment of severity of symptoms and functional status in carpal tunnel syndrome. J Bone Joint Surg Am. 1993;75(11):1585-92.

52. Atroshi I, Lyren PE, Ornstein E, Gummesson C. The six-item CTS symptoms scale and palmar pain scale in carpal tunnel syndrome. J Hand Surg. 2011;36(5):788-94

53. Sangha O, Stucki G, Liang MH, Fossel AH, Katz JN. The self-administered comorbidity questionnaire: a new method to assess comorbidity for clinical and health services research. Arthritis Rheum. 2003;49(2):156-63.

54. Bland JD. A neurophysiological grading scale for carpal tunnel syndrome. Muscle Nerve. 2000;23(8):1280-3.

55. Treede RD, Jensen TS, Campbell JN, Cruccu G, Dostrovsky JO, Griffin JW, et al. Neuropathic pain: redefinition and a grading system for clinical and research purposes. Neurology. 2008;70(18):1630-5.

56. Edwards RR, Dworkin RH, Turk DC, Angst MS, Dionne R, Freeman R, et al. Patient phenotyping in clinical trials of chronic pain treatments: IMMPACT recommendations. Pain. 2016;157(9):1851-71.

57. Baron R, Maier C, Attal N, Binder A, Bouhassira D, Cruccu G, et al. Peripheral neuropathic pain: a mechanism-related organizing principle based on sensory profiles. Pain. 2017;158(2):261-72.

58. Bouhassira D, Attal N, Alchaar H, Boureauc F, Brochet B, Bruxelle J, et al. Comparison of pain syndromes associated with nervous or somatic lesions and development of a new neuropathic pain diagnostic questionnaire (DN4). Pain. 2005;114:29-36.

59. Bouhassira D, Attal N, Fermanian J, Alchaar H, Gautron M, Masquelier E, et al. Development and validation of the neuropathic pain symptom inventory. Pain. 2004;108(3):248-57.

60. Freeman R, Baron R, Bouhassira D, Cabrera J, Emir B. Sensory profiles of patients with neuropathic pain based on the neuropathic pain symptoms and signs. Pain. 2014;155(2):367-76.

61. Tan G, Jensen MP, Thornby Jl, Shanti BF. Validation of the brief pain inventory for chronic nonmalignant pain. J Pain. 2004;5(2):133-7.

62. Cleeland CS. Measurement of pain by subjective report. In: Chapman CR, Chapman LJ, editors. Advances in Pain Research and Therapy. 12: Issues in Pain Measurement. New York: Raven Press; 1989. p. 391-403.

63. Kapstad H, Hanestad BR, Langeland N, Rustoen T, Stavem K. Cutpoints for mild, moderate and severe pain in patients with osteoarthritis of the hip or knee ready for joint replacement surgery. BMC Musculoskelet Disord. 2008:9:55.

64. Boonstra AM, Schiphorst Preuper HR, Balk GA, Stewart RE. Cut-off points for mild, moderate, and severe pain on the visual analogue scale for pain in patients with chronic musculoskeletal pain. Pain. 2014;155(12):2545-50.

65. De Carvalho Leite JC, Jerosch-Herold C, Song F. A systematic review of the psychometric properties of the Boston carpal tunnel questionnaire. BMC Musculoskelet Disord. 2006;7:78.

66. Jerosch-Herold C, Shepstone L, Miller L, Chapman P. The responsiveness of sensibility and strength tests in patients undergoing carpal tunnel decompression. BMC Musculoskelet Disord. 2011;12:244.

67. Sullivan MJL, Bishop SR. The pain Catastrophizing scale: development and validation. Psychol Assess. 1995;7(4):524-32.

68. Keefe FJ, Rumble ME, Scipio CD, Giordano LA, Perri LM. Psychological aspects of persistent pain: current state of the science. J Pain. 2004;5(4):195-211.

69. Crombez G, De Paepe AL, Veirman E, Eccleston C, Verleysen G, Van Ryckeghem DML. Let's talk about pain catastrophizing measures: an item content analysis. PeerJ. 2020;8:e8643.

70. Pincus T, Williams AC, Vogel S, Field A. The development and testing of the depression, anxiety, and positive outlook scale (DAPOS). Pain. 2004;109(1-2):181-8

71. Pincus T, Rusu A, Santos R. Responsiveness and construct validity of the depression, anxiety, and positive outlook scale (DAPOS). Clin J Pain. 2008;24(5):431-7.

72. Morin $\mathrm{CM}$, Belleville $\mathrm{G}$, Belanger $\mathrm{L}$, Ivers $\mathrm{H}$. The insomnia severity index: psychometric indicators to detect insomnia cases and evaluate treatment response. Sleep. 2011;34(5):601-8.

73. Olejnik S, Algina J. Measures of effect size for comparative studies: applications, interpretations, and limitations. Contemp Educ Psychol. 2000;25(3):241-86.

74. Cohen J. Statistical power analysis for the behavioral sciences. 2nd ed. Hillsdale: Lawrence Erlbaum Associates; 1988.

75. Baskozos G, Sandy-Hindmarch O, Clark AJ, Windsor K, Karlsson P, Weir GA, et al. Molecular and cellular correlates of human nerve regeneration: ADCYAP1/PACAP enhance nerve outgrowth. Brain. 2020;143(7):2009-26.

76. Atroshi I, Gummesson C, Johnsson R, Sprinchorn A. Symptoms, disability, and quality of life in patients with carpal tunnel syndrome. J Hand Surg. 1999;24(2):398-404.

77. Gursoy AE, Kolukisa M, Yildiz GB, Kocaman G, Celebi A, Kocer A Relationship between electrodiagnostic severity and neuropathic pain assessed by the LANSS pain scale in carpal tunnel syndrome. Neuropsychiatr Dis Treat. 2013;9:65-71.

78. Turgut ST, İçağasıoğlu A, Selimoğlu E, Atlığ RŞ, Adatepe T, Mesci E. The Relationship Between Electrodiagnostic Findings and the DN4 Questionnaire in Patients with Carpal Tunnel Syndrome. J Musculoskelet Pain. 2013:21(1):19-22.

79. Truini A, Padua L, Biasiotta A, Caliandro P, Pazzaglia C, Galeotti F, et al. Differential involvement of A-delta and A-beta fibres in neuropathic pain related to carpal tunnel syndrome. Pain. 2009;145(1-2):105-9.

80. Han T, Bai J, Liu W, Hu Y. A systematic review and meta-analysis of alphalipoic acid in the treatment of diabetic peripheral neuropathy. Eur J Endocrinol. 2012;167(4):465-71.

81. Pajardi G, Bortot P, Ponti V, Novelli C. Clinical usefulness of oral supplementation with alpha-lipoic acid, curcumin phytosome, and B-group vitamins in patients with carpal tunnel syndrome undergoing surgical treatment. Evid Based Complement Alternat Med. 2014;2014:891310.

82. Boriani F, Granchi D, Roatti G, Merlini L, Sabattini T, Baldini N. Alphalipoic acid after median nerve decompression at the carpal tunnel: a randomized controlled trial. J Hand Surg. 2017:42(4):236-42.

83. Monroy Guizar EA, Garcia Benavides L, Ambriz Plascencia AR, Pascoe Gonzalez S, Totsuka Sutto SE, Cardona Munoz EG, et al. Effect of alphaLipoic acid on clinical and neurophysiologic recovery of carpal tunnel syndrome: a double-blind, Randomized Clinical Trial. J Med Food. 2018;21(5):521-6.

84. Passiatore M, Perna A, De-Vitis R, Taccardo G. The use of Alfa-Lipoic acid-R (ALA-R) in patients with mild-moderate carpal tunnel syndrome: a randomised controlled open label prospective study. Malays Orthop J. 2020;14(1):1-6. 
85. Quartana PJ, Campbell CM, Edwards RR. Pain catastrophizing: a critical review. Expert Rev Neurother. 2009;9(5):745-58.

86. O'Brien J. Calls to Rename "Pain Catastrophizing" Backed by International Patient-Researcher Partnership Washington, D.C.: International Association for the Study of Pain; 2020 [Available from: https://www. painresearchforum.org/news/150718-calls-rename-\%E2\%80\%9Cpaincatastrophizing\%E2\%80\%9D-backed-international-patient-researcherpartnership

87. Wade JB, Riddle $D L$, Thacker $L R$. Is pain catastrophizing a stable trait or dynamic state in patients scheduled for knee arthroplasty? Clin J Pain. 2012;28(2):122-8.

88. Mosegaard SB, Stilling M, Hansen TB. Higher preoperative pain catastrophizing increases the risk of low patient reported satisfaction after carpal tunnel release: a prospective study. BMC Musculoskelet Disord. 2020;21(1):42

89. Egbert LD, Battit $G$, Turndorf $H$, Beecher HK. The value of the preoperative visit by an anesthetist. A study of doctor-patient rapport. JAMA. 1963;185(7):553-5.

90. Ramsay MA. A survey of pre-operative fear. Anaesthesia. 1972:27(4):396-402.

91. Stamenkovic DM, Rancic NK, Latas MB, Neskovic V, Rondovic GM, Wu JD, et al. Preoperative anxiety and implications on postoperative recovery: what can we do to change our history. Minerva Anestesiol. 2018;84(11):1307-17.

92. Pritchard MJ. Managing anxiety in the elective surgical patient. Br J Nurs. 2009;18(7):416-9.

93. Afolalu F, Ramlee F, Tang N. Effects of sleep changes on pain-related health outcomes in the general population : a systematic review of longitudinal studies with exploratory meta-analysis. Sleep Med Rev. 2017;(39):82-97.

94. Lautenbacher S, Kundermann B, Krieg JC. Sleep deprivation and pain perception. Sleep Med Rev. 2006;10(5):357-69.
95. Simpson NS, Scott-Sutherland J, Gautam S, Sethna N, Haack M. Chronic exposure to insufficient sleep alters processes of pain habituation and sensitization. Pain. 2018;159(1):33-40.

96. Bianchi MT, Williams KL, McKinney S, Ellenbogen JM. The subjectiveobjective mismatch in sleep perception among those with insomnia and sleep apnea. J Sleep Res. 2013;22(5):557-68.

97. Boya H, Ozcan O, Oztekin HH. Long-term complications of open carpal tunnel release. Muscle Nerve. 2008;38(5):1443-6.

98. Citron ND, Bendall SP. Local symptoms after open carpal tunnel release. A randomized prospective trial of two incisions. J Hand Surg. 1997;22(3):317-21.

99. Povlsen B, Tegnell L, Revell M, Adolfsson L. Touch allodynia following endoscopic (single portal) or open decompression for carpal tunnel syndrome. J Hand Surg. 1997;22(3):325-7.

100. Kaczkurkin AN, Foa EB. Cognitive-behavioral therapy for anxiety disorders: an update on the empirical evidence. Dialogues Clin Neurosci. 2015;17(3):337-46.

101. Schmid AB, Bland JD, Bhat MA, Bennett DL. The relationship of nerve fibre pathology to sensory function in entrapment neuropathy. Brain. 2014:137(Pt 12):3186-99.

102. Gerritsen AA, de Krom MC, Struijs MA, Scholten RJ, de Vet HC, Bouter LM. Conservative treatment options for carpal tunnel syndrome: a systematic review of randomised controlled trials. J Neurol. 2002;249(3):272-80

\section{Publisher's Note}

Springer Nature remains neutral with regard to jurisdictional claims in published maps and institutional affiliations.
Ready to submit your research? Choose BMC and benefit from:

- fast, convenient online submission

- thorough peer review by experienced researchers in your field

- rapid publication on acceptance

- support for research data, including large and complex data types

- gold Open Access which fosters wider collaboration and increased citations

- maximum visibility for your research: over $100 \mathrm{M}$ website views per year

At $\mathrm{BMC}$, research is always in progress.

Learn more biomedcentral.com/submissions 